\title{
Performance of van der Waals Corrected Functionals for Guest Adsorption in the $\mathbf{M}_{\mathbf{2}}$ (dobdc) Metal-Organic Frameworks
}

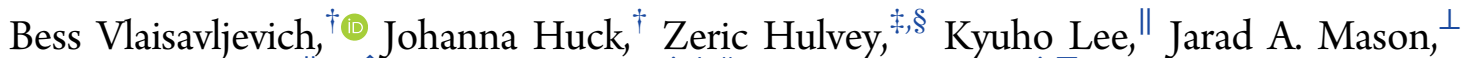
Jeffrey B. Neaton, $\|, \bullet, \diamond$ Jeffrey R. Long, ${ }^{\dagger, \perp, \#}$ Craig M. Brown, ${ }^{\ddagger}, \nabla$ Dario Alfè, ${ }^{\bigcirc}$ Angelos Michaelides, $\bullet$ and Berend Smit ${ }^{*},+, I_{\odot}$

${ }^{\dagger}$ Department of Chemical and Biomolecular Engineering, ${ }^{\perp}$ Department of Chemistry, and $\bullet$ Department of Physics, University of California, Berkeley, California 94720, United States

${ }^{\ddagger}$ Center for Neutron Research, National Institute of Standards and Technology, Gaithersburg, Maryland 20899, United States

${ }^{\S}$ Department of Materials Science and Engineering, University of Maryland, College Park, Maryland 20742 United States

"Molecular Foundry and "Materials Science Division, Lawrence Berkeley National Laboratory, Berkeley, California 94720 , United States

${ }^{\nabla}$ Department of Chemical and Biomolecular Engineering, University of Delaware, Newark, Delaware 19716, United States

ODepartment of Earth Sciences, Thomas Young Centre and London Centre for Nanotechnology, University College London, Gower Street, London WC1E 6BT, United Kingdom

London Centre for Nanotechnology and Department of Physics and Astronomy, Thomas Young Centre, University College London, Gower Street, London WC1E 6BT, United Kingdom

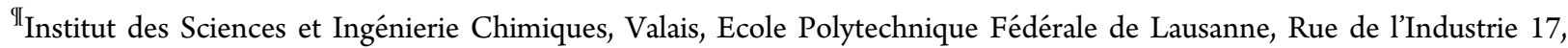
CH-1951 Sion, Switzerland

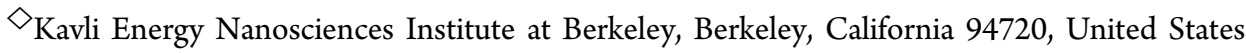

Supporting Information

ABSTRACT: Small-molecule binding in metal-organic frameworks (MOFs) can be accurately studied both experimentally and computationally, provided the proper tools are employed. Herein, we compare and contrast properties associated with guest binding by means of density functional theory (DFT) calculations using nine different functionals for the $\mathrm{M}_{2}$ (dobdc) (dobdc ${ }^{4-}=$ 2,5-dioxido,1,4-benzenedicarboxylate) series, where $\mathrm{M}=\mathrm{Mg}, \mathrm{Mn}$, $\mathrm{Fe}, \mathrm{Co}, \mathrm{Ni}, \mathrm{Cu}$, and $\mathrm{Zn}$. Additionally, we perform Quantum Monte Carlo (QMC) calculations for one system to determine if this method can be used to assess the performance of DFT. We also make comparisons with previously published experimental results for carbon dioxide and water and present new methane neutron powder diffraction (NPD) data for further comparison.

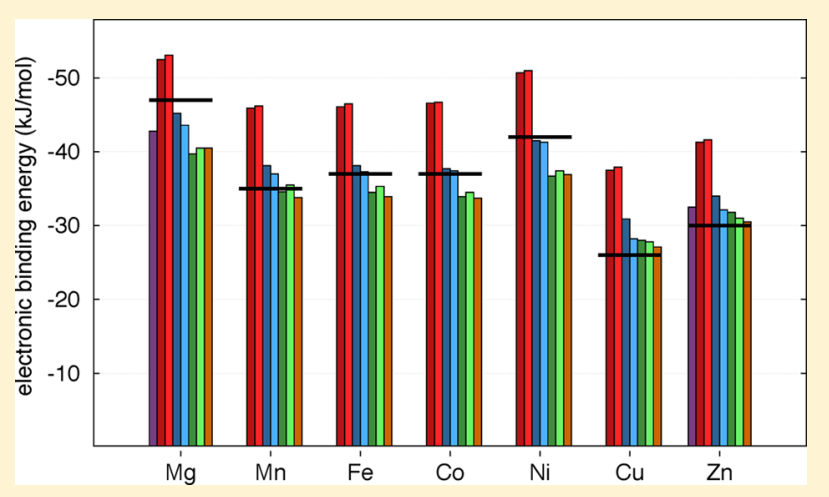
All of the functionals are able to predict the experimental variation in the binding energy from one metal to the next; however, the interpretation of the performance of the functionals depends on which value is taken as the reference. On the one hand, if we compare against experimental values, we would conclude that the optB86b-vdW and optB88-vdW functionals systematically overestimate the binding strength, while the second generation of van der Waals (vdW) nonlocal functionals (vdw-DF2 and revvdW-DF2) correct for this providing a good description of binding energies. On the other hand, if the QMC calculation is taken as the reference then all of the nonlocal functionals yield results that fall just outside the error of the higher-level calculation. The empirically corrected vdW functionals are in reasonable agreement with experimental heat of adsorptions but under bind when compared with QMC, while Perdew-Burke-Ernzerhof fails by more than $20 \mathrm{~kJ} / \mathrm{mol}$ regardless of which reference is employed. All of the functionals, with the exception of vdW-DF2, predict reasonable framework and guest binding geometries when compared with NPD measurements. The newest of the functionals considered, rev-vdW-DF2, should be used in place of vdWDF2, as it yields improved bond distances with similar quality binding energies.

\section{INTRODUCTION}

Metal-organic frameworks (MOFs) are highly promising materials for gas storage and gas separations due to their crystallinity, high porosity, and tunability. Indeed, the vast number of

Received: January 3, 2017

Revised: April 21, 2017

Published: April 24, 2017 
combinations of organic linkers and metal connectors that can be employed allow one to, in principle, design materials for a wide variety of potential applications. ${ }^{1,2}$ Unlike other classes of porous materials, MOFs can be synthesized and designed using insights from coordination chemistry. In recent years, a wide variety of coordination solids and MOFs have been made. In many cases, the goal is to design materials with binding pockets tuned to selectively bind one guest (e.g., storage applications) or that favor the adsorption of one guest over another (e.g., separations). From this perspective, it is essential to understand how the framework and the guest interact. Diffraction experiments and simulation can play an important role in developing this understanding. ${ }^{3}$

Classical methods have played a crucial role in this regard. Gas adsorption can be modeled using Monte Carlo simulations where thermodynamic properties (most often the heat of adsorption and the loading of guest molecules at a given temperature and pressure) are calculated as an ensemble average. ${ }^{4,5}$ This is particularly important, since gas molecules generally physisorb in the MOF and at finite temperatures can access many binding configurations. Additionally, molecular dynamics simulations have been applied to study the framework and the motion of small molecules in MOFs, including the calculation of diffusion constants. ${ }^{6,7}$ Classical force fields have been developed specifically for treating guest binding in MOFs and for the bonding interactions within the frameworks. ${ }^{4,8}$ These force fields as well as semiempirical methods have been used to optimize framework geometries, particularly when breathing motions in MOFs are important or when a large database of MOFs is studied and optimizations with higher levels of theory are intractable. ${ }^{9-11}$

However, electronic structure methods are best suited to study a smaller number of materials with a focus on understanding the molecular level details of guest binding at the primary binding site (or sites) in MOFs. The ability of density functional theory (DFT) to balance accuracy and computational efficiency has led to its widespread use in electronic structure theory, whether for molecular systems, solids, surfaces, or in this case porous materials. Given the large size of the unit cells of most MOFs, DFT is the quantum chemical method most suitable for routine calculations on the periodic structure of MOFs. However, as is always the case with DFT, the accuracy depends on the exchange-correlation functional that is employed.

In porous materials where guest molecules generally interact with the surface through physisorption, dispersion interactions can dominate substrate binding. A well-known drawback of conventional Local Density Approximation (LDA) or Generalized Gradient Approximation (GGA) functionals is their inability to describe properly the London dispersion forces that originate from nonlocal electron correlation. ${ }^{12-17}$ Fortunately, much work has been accomplished in the DFT community to properly account for such weak interactions using a variety approaches. $^{12-17}$ One class of functionals is the van der Waals (vdW) inclusive functional developed originally by Langreth, Lunqvist, and co-workers (the so-called DF functionals) and the second-generation version of this functional (the so-called DF2 functionals). ${ }^{18,19} \mathrm{~A}$ second class of functionals are those that account for dispersion forces through empirical van der Waals correction approaches (the so-called $-\mathrm{D}$ Functionals), such as those developed by Grimme and co-workers, and a third class are certain parametrized meta-GGA functionals that have been shown to recover dispersion. ${ }^{5,20}$ MOFs have been successfully studied, for a variety of applications, using each of these three classes of functionals (DF/DF2, $-\mathrm{D}$, and meta-GGA).
However, in most studies the chemistry of the specific materials being considered has been the main focus rather than understanding the performance of the various DFT functionals used. Therefore, in the current study we chose a set of functionals to be representative of these three classes of functionals and focused on understanding their performance in terms of binding for a set of small molecules. There are, of course, many other promising functionals that have been developed to treat dispersion interactions, and so our study is by no means allinclusive. $^{12-17,21-23}$

Few MOFs are as well-studied, both computationally and experimentally, as the $\mathrm{M}_{2}$ (dobdc) family (Figure 1 ), which is

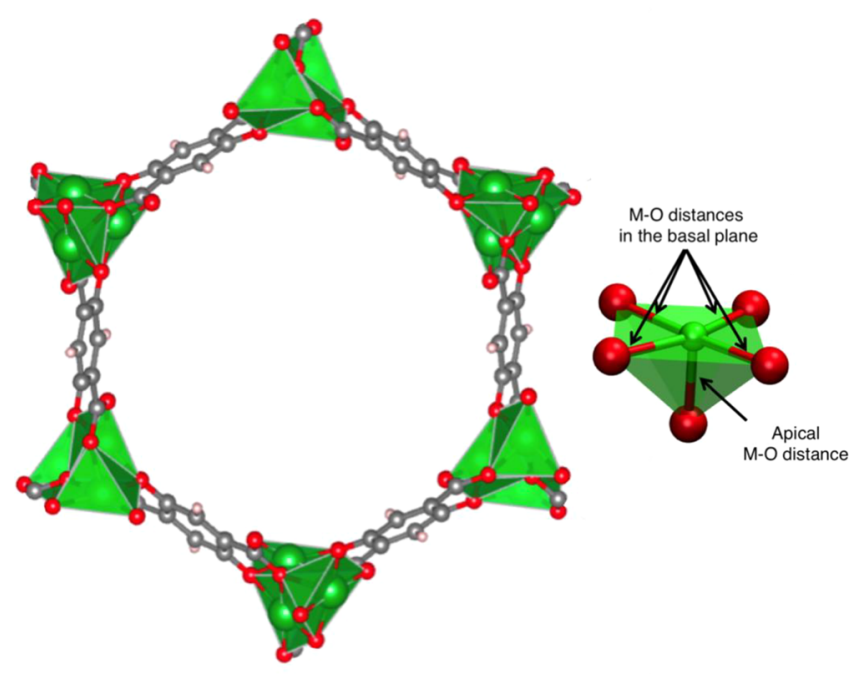

Figure 1. Hexagonal channel of $M_{2}$ (dobdc) where the metal $(M=M g$, $\mathrm{Mn}, \mathrm{Fe}, \mathrm{Co}, \mathrm{Ni}, \mathrm{Cu}$, and $\mathrm{Zn}$ ) is shown in green with a polyhedral representation, carbon is shown in gray, oxygen in red, and hydrogen in white. (inset) The apical $\mathrm{M}-\mathrm{O}$ bond and the $\mathrm{M}-\mathrm{O}$ bonds in the basal plane.

also referred to as M-MOF-74, CPO-27-M, or $\mathrm{M}_{2}$ (dhtp), where $\mathrm{M}=\mathrm{Mg}, \mathrm{Mn}, \mathrm{Fe}, \mathrm{Co}, \mathrm{Ni}, \mathrm{Cu}$, or $\mathrm{Zn} .^{24-51}$ These frameworks have open metal sites to which adsorbates can bind strongly, allowing the effect of changing the metal to be studied systematically. Recently, the manner in which a wide variety of small molecules bind in $\mathrm{M}_{2}$ (dobdc) was studied with DFT for first-row transition metals, for both the experimentally characterized materials $(\mathrm{M}=\mathrm{Mg}, \mathrm{Mn}, \mathrm{Fe}, \mathrm{Co}, \mathrm{Ni}, \mathrm{Cu}$, or $\mathrm{Zn})$ and some that have not yet been realized $(\mathrm{M}=\mathrm{Ti}, \mathrm{V}$, or $\mathrm{Cr}){ }^{33}$ In this earlier study by Lee et al., ${ }^{33}$ it was noted that, while many $\mathrm{M}_{2}(\mathrm{dobdc})$ compounds have been investigated computationally, several levels of theory have been employed, making a direct comparison between studies difficult. Specifically, Lee et al. optimized the frameworks using the Perdew-Burke-Ernzerhof (PBE) functional, ${ }^{52}$ while the vdW-DF2 functional ${ }^{19}$ was employed to treat the weak binding of a series of guests with the framework for all metal cations $\mathrm{M}$. Their aim was to develop a comprehensive data set as a basis for future comparisons. While this previous study by some of the authors used one level of theory to understand how guest binding changes across a spectrum of small molecules, in this work, we focus on how a select number of density functionals perform for three guests, namely, $\mathrm{H}_{2} \mathrm{O}, \mathrm{CO}_{2}$, and $\mathrm{CH}_{4}-$ molecules for which several other groups have computed adsorption energies in the $\mathrm{M}_{2}$ (dobdc) series. For example, Lee et al. and Canepa et al. studied water adsorption, ${ }^{33,34}$ while Lee et al., Canepa et al., and Rana et al. studied methane adsorption with 
nonlocal density functionals. ${ }^{33-35}$ Sillar et al. studied methane adsorption using the so-called hybrid MP2/cbs:DFT+D + $\Delta \operatorname{CCSD}(\mathrm{T})$ approach, ${ }^{36}$ where binding energies are computed using periodic empirical dispersion-corrected DFT (a -D functional) but are corrected by second-order Møller-Plesset perturbation theory (MP2) calculations and coupled cluster calculations with single and double excitations and perturbative treatment of triple excitations $(\mathrm{CCSD}(\mathrm{T}))$ on finite-sized models. $\mathrm{CO}_{2}$ is by far the most studied guest in the $\mathrm{M}_{2}$ (dobdc) framework with a wide variety of density functionals. ${ }^{33,34,37-45}$ Cluster models have also been used to study both $\mathrm{CO}_{2}$ and $\mathrm{CH}_{4}{ }^{46-49}$ Overall, what is most notable when looking at the data in the literature is the deviations among the reported energies. For example, the $\mathrm{CO}_{2}$ binding energies reported for $\mathrm{Mg}_{2}$ (dobdc), excluding data for GGAs without vdW corrections, range from 41.2 to $62.1 \mathrm{~kJ} / \mathrm{mol}$ for electronic binding energies and from 37.4 to $58.3 \mathrm{~kJ} / \mathrm{mol}$ for enthalpies. ${ }^{37,38}$

Experimental data can provide a reference for calculated energies and structures. In this work, we use published heat of adsorption data as a reference for DFT energies and published neutron powder diffraction (NPD) data as a reference for the DFT-optimized framework geometries as well as the geometries of $\mathrm{CO}_{2}$ and $\mathrm{H}_{2} \mathrm{O}$ bound in the MOF. For $\mathrm{CH}_{4}$, no NPD data was available and was generated as part of this work. Comparisons with experiment are very helpful, but we wish to emphasize that challenges in the characterization of the materials, structural defects, and thermal effects can make the comparison between experiment and simulation indirect. ${ }^{3}$ Therefore, high-level Quantum Monte Carlo (QMC) calculations were also performed to study $\mathrm{CO}_{2}$ binding in the $\mathrm{Fe}_{2}$ (dobdc) framework with the goal of evaluating the utility QMC as a high-level reference for the performance of the various DFT functionals. In the following, we will show the performance of nine functionals for three guests $\left(\mathrm{CH}_{4}, \mathrm{CO}_{2}\right.$, and $\left.\mathrm{H}_{2} \mathrm{O}\right)$ that were chosen for this study, since they represent a range of binding strengths.

\section{COMPUTATIONAL DETAILS}

2.1. Periodic Density Functional Theory. In this work, we compare the performance of eight vdW density functionals and the PBE functional, as an example of a standard GGA, ${ }^{52}$ for smallmolecule binding in the $\mathrm{M}_{2}$ (dobdc) framework. This framework consists of a divalent metal connector $(\mathrm{M}=\mathrm{Mg}, \mathrm{Mn}, \mathrm{Fe}, \mathrm{Co}, \mathrm{Ni}$, $\mathrm{Cu}$, or $\mathrm{Zn}$ ) and 2,5-dioxido-1,4-benzenedicarboxylate (dobdc ${ }^{4-}$ ) as the linker (see Figure 1). ${ }^{24-32}$ Specifically, we will explore the performance of the DF functionals: optB88-vdW $\mathrm{dW}^{18,53}$ and optB86b-vdW; ${ }^{18,54}$ the DF2 functionals: vdW-DF2 ${ }^{19}$ and rev-vdW-DF2; ${ }^{19,55}$ the $-D$ functionals: PBE-D2, ${ }^{52,56}$ PBE$\mathrm{D} 3{ }^{52,57}$ and PBE-D3 BJ; ${ }^{52,57,58}$ and one meta-GGA functional: M06-L. ${ }^{59,60}$ Although all belong to the group of GGA functionals, they generally differ in the nature of the correction that is additionally integrated to include London dispersion interactions. As an aside, the vdW-DF2 functional is known to overestimate bond and lattice distances; ${ }^{54}$ therefore, the rev-vdWDF2 functional was recently developed by Hamada with the purpose of improving the geometries of $\mathrm{vdW}-\mathrm{DF} 2 .{ }^{55}$ All periodic DFT calculations were performed with the Vienna Ab Initio Simulation Package (VASP) with a plane-wave basis set and projector augmented wave (PAW) potentials, where $\mathrm{C}(2 \mathrm{~s} 2 \mathrm{p})$, $\mathrm{O}(2 \mathrm{~s} 2 \mathrm{p}), \mathrm{Mg}$ (3s), Mn(4s3d), Fe (3d), Co (4s3d), Ni (4s3d), $\mathrm{Cu}(4 \mathrm{p} 3 \mathrm{~d})$, and $\mathrm{Zn}(4 \mathrm{~s} 3 \mathrm{~d})$ electrons were included explicitly in the valence. ${ }^{61}$ For the calculations performed on $\mathrm{Fe}_{2}$ (dobdc) with the PBE-D3-based functionals, the semicore $\mathrm{p}$-states were also treated as valence states, $\mathrm{Fe}(4 \mathrm{~s} 3 \mathrm{~d} 3 \mathrm{p})$.
The primitive rhombohedral unit cell containing 54 atoms was used for all calculations (optimized geometries are included as Supporting Information), and on-site Hubbard $U$ corrections were employed for metal d electrons. ${ }^{62}$ It has been previously reported that the so-called $U$ correction is required for transition metals with unpaired electrons in $\mathrm{M}_{2}$ (dobdc) to obtain trends in binding enthalpies observed experimentally. ${ }^{33,63}$ While $U$ values can also be determined from first principles, ${ }^{63}$ we used values of $U$ determined to reproduce oxidation energies in the respective metal oxides. ${ }^{64}$ The specific values used were: $3.8,4.0,3.3,6.4$, and $3.8 \mathrm{eV}$ for $\mathrm{Mn}, \mathrm{Fe}, \mathrm{Co}, \mathrm{Ni}$, and $\mathrm{Cu}$, respectively. ${ }^{64}$ No $U$ correction was applied for $\mathrm{Mg}$ and $\mathrm{Zn}$, since they do not have unpaired electrons.

In Section 4.1, binding energy calculations are reported for $\mathrm{CO}_{2}$ in the $\mathrm{Fe}_{2}$ (dobdc) framework for the eight vdW corrected functionals and PBE. The binding energies were computed at the geometry optimized in Lee et al. ${ }^{33}$ Electronic binding energies $\left(\Delta E_{\mathrm{BE}}\right)$ were calculated as

$$
\Delta E_{\mathrm{BE}}=E_{\text {guest }+\mathrm{MOF}}-E_{\mathrm{MOF}}-E_{\text {guest }}
$$

where $E_{\text {guest+MOF }}$ is the total energy of the framework and bound guest, $E_{\mathrm{MOF}}$ is the total energy of the MOF, and $E_{\text {guest }}$ is the total energy of the guest. The single-point energy calculations used in the binding energy calculations were performed with a $1000 \mathrm{eV}$ cutoff.

In Section 4.2, the atomic positions and lattice constants for the empty frameworks were optimized with forces converged to $0.02 \mathrm{eV} / \AA$ f for all metals and all functionals. A cutoff in the planewave basis of $550 \mathrm{eV}$ was used along with the $\boldsymbol{\Gamma}$-Point sampling of reciprocal space. The use of this cutoff for framework optimizations was tested for the optB88-vdW functional by performing geometry optimizations with higher cutoff of $1000 \mathrm{eV}$. The higher cutoff resulted in a change of at most $0.008 \AA$ in lattice constants. Furthermore, when employing a $2 \times 2 \times 2$ MonkhorstPack $k$-point mesh the lattice parameters of the empty framework changed by at most $0.03 \AA$ A. See Table S12 for the results of this test.

In Section 4.3, binding energies were computed for the three guests. For $\mathrm{CO}_{2}$ and $\mathrm{CH}_{4}$, the rigid framework approach was applied, and therefore the framework geometries were fixed at the PBE optimized geometry ${ }^{52}$ (structures previously reported by Lee et al. are used herein). ${ }^{33}$ Rigid framework approximations can be applied in MOFs, not only in DFT geometry optimizations but also in molecular simulations. Strong guest binding energies or framework flexibility are signs that this approximation could break down, and its use should be tested. To test the validity of the rigid framework approximation in this case, a full geometry optimization was performed using the optB88-vdW functional (see Table S10). The $\mathrm{CO}_{2}$ and $\mathrm{CH}_{4}$ interaction is sufficiently weak that the framework geometry and binding energies remain largely unaltered when the constraint of the rigid framework approximation is removed leading to $\mathrm{CO}_{2}$ and $\mathrm{CH}_{4}$ binding energies in the $\mathrm{Mg}_{2}$ (dobdc) framework changing by only 2.5 and $1.1 \mathrm{~kJ} / \mathrm{mol}$, respectively. On the one hand, this is further supported by the experimental work of Queen et al. that has shown that volume changes are less than $0.5 \%$ upon $\mathrm{CO}_{2}$ adsorption for all materials characterized to date. ${ }^{43}$ On the other hand, $\mathrm{H}_{2} \mathrm{O}$ binds more strongly, and the rigid framework approximation breaks down (compare Tables S4 and S11); therefore, the binding energies reported in this section for $\mathrm{H}_{2} \mathrm{O}$ result from full geometry optimizations (lattice and atoms).

Initial guesses for guest binding sites were taken from Lee et al. ${ }^{33}$ Subsequently, the position of the guest (or for $\mathrm{H}_{2} \mathrm{O}$ 
all atoms) was optimized with each functional using a plane-wave cutoff of $550 \mathrm{eV}$ with forces converged to $0.02 \mathrm{eV} / \AA ̊$. Singlepoint energy calculations with a higher cutoff of $1000 \mathrm{eV}$ were used when computing the electronic binding energies. The $\boldsymbol{\Gamma}$-point sampling of reciprocal space was used in all calculations. Tests were again performed with the optB88-vdW functional. The effect of changing the $k$-point mesh (Table S13) and the cutoff (Tables S13-S16) on the electronic binding energy are given as Supporting Information. The effect is within a kilojoule per mole, with the one exception of PBE-D3; therefore, the higher cutoff $1000 \mathrm{eV}$ will only be used in optimizations with the PBE-D3 functionals. Finally, M06-L calculations were only performed for $\mathrm{Mg}$ and $\mathrm{Zn}$ (closed-shell cases), since the selfconsistent field (SCF) convergence in VASP is challenging, and the calculations are time intensive.

Also in Section 4.3, the heat of adsorption values measured experimentally are enthalpies, but electronic binding energies were calculated. To calculate the heat of adsorption, thermal corrections were computed, and these corrections were $\sim 4 \mathrm{~kJ} / \mathrm{mol}$ for $\mathrm{CO}_{2}, 5 \mathrm{~kJ} / \mathrm{mol}$ for $\mathrm{CH}_{4}$, and $6 \mathrm{~kJ} / \mathrm{mol}$ for $\mathrm{H}_{2} \mathrm{O}$ (see Table $\mathrm{S} 17$ for exact values). To make the comparison between experiment and theory, we subtracted the vdW-DF2 calculated vibrational correction (computed in the work of Lee et $\mathrm{al}^{33}$ ) from the experimental heat of adsorption. We tested the sensitivity of the vibrational contribution with respect to the functional for $\mathrm{CO}_{2}$ in the $\mathrm{Mg}_{2}$ (dobdc) framework and found that it varied by only $0.1 \mathrm{~kJ} / \mathrm{mol}$ (see Table S18).

2.2. Quantum Monte Carlo. Diffusion Monte Carlo calculations (referred to throughout simply as QMC) were performed using the CASINO code. ${ }^{65}$ The DFT-optimized structures from Lee et al. ${ }^{33}$ were used along with trial wave functions of the Slater-Jastrow type:

$$
\Psi_{T}(\mathbf{R})=\mathbf{D}^{\uparrow} \mathbf{D}^{\downarrow} e^{\mathrm{J}}
$$

where $\mathbf{D}^{\uparrow}$ and $\mathbf{D}^{\downarrow}$ are Slater determinants of up- and down-spin single-electron orbitals. The Jastrow factor $e^{J}$ is the exponential of a sum of one-body, two-body, and three-body terms, which are parametrized functions of electron-nucleus, electron-electron, and electron-electron-nucleus separations, respectively, and are designed to satisfy the cusp conditions. The parameters in the Jastrow factor were varied to minimize the variance of the local energy $E_{L}(\mathbf{R})=\Psi_{\mathrm{T}}^{-1}(\mathbf{R}) \hat{H} \Psi_{\mathrm{T}}(\mathbf{R})$. Imaginary time evolution of the Schrödinger equation was performed with the usual short time approximation and the recently proposed modifications of the Green Function ${ }^{66}$ using time steps $\mathrm{d} t=0.005,0.01,0.02$, 0.05 , and $0.1 \mathrm{au}$, which showed convergence of the binding energy to within the statistical error of $2 \mathrm{~kJ} / \mathrm{mol}$ at $\mathrm{d} t=0.02 \mathrm{au}$. We used Dirac-Fock pseudopotentials $(\mathrm{PP})^{67}$ for $\mathrm{C}, \mathrm{H}$, and $\mathrm{O},{ }^{68}$ and a DFT norm-conserving PP for Fe. ${ }^{69}$ To treat the nonlocal part of the pseudopotential, we used both the locality approximation $^{66}$ and the T-move method, ${ }^{70}$ which gave binding energies agreeing with each other within the statistical error of $2 \mathrm{~kJ} / \mathrm{mol}$. The single-particle orbitals were obtained with DFT-LDA calculations using a plane-wave basis with a single Brillouin zone-boundary point using the PWSCF package. ${ }^{71} \mathrm{We}$ used a plane-wave cutoff of $600 \mathrm{Ry}(8163 \mathrm{eV})$ and re-expanded the single particle orbitals in terms of $B$ splines ${ }^{72}$ using the natural $B$-spline grid spacing given by $a=\pi / G_{\max }$, where $G_{\max }$ is the length of the largest plane wave. The Diffusion Monte Carlo (DMC) calculations were performed using the Ewald technique to model electron-electron interactions. Finite size corrections according Chiesa et al. ${ }^{73}$ had a negligible effect on the $\mathrm{CO}_{2}$ binding energy, while those according to the Model Periodic
Coulomb interactions gave a correction of $-3 \mathrm{~kJ} .{ }^{74}$ The number of walkers in the DMC simulations was 20480 for the $\mathrm{CO}_{2}$ molecule and 90000 for the MOF and $\mathrm{MOF}-\mathrm{CO}_{2}$ systems.

\section{EXPERIMENTAL DETAILS}

3.1. Synthesis. All reagents were obtained from commercial vendors and used without further purification. Samples of all of the $\mathrm{M}_{2}$ (dobdc) materials were synthesized according to literature procedures. $^{24,30,75-77}$ The successful synthesis and activation of each compound was confirmed by comparing the X-ray powder diffraction patterns and Langmuir surface areas to those previously reported.

3.2. Neutron Diffraction. NPD experiments were performed on activated $\mathrm{M}_{2}$ (dobdc) samples using the high-resolution neutron powder diffractometer BT-1 at the National Institute of Standards and Technology Center for Neutron Research (NCNR). Between 0.7 and $4.0 \mathrm{~g}$ of the materials were placed in a helium glovebox and loaded into vanadium sample cells equipped with a valve for gas loading and sealed using an indium O-ring. Data were collected using a $\mathrm{Ge}(311)$ monochromator with an in-pile $60^{\prime}$ collimator corresponding to a wavelength of $2.078 \AA$. The samples were loaded onto bottom-loading closedcycle refrigerators and initially cooled to $150 \mathrm{~K}$. The samples were then connected through the gas loading valve to a manifold of known volume and exposed to a quantitative dose of $\mathrm{CD}_{4}$ corresponding to $0.75 \mathrm{CD}_{4}$ molecules per metal atom. Deuterated methane was used in these experiments due to the large incoherent scattering cross section of hydrogen, which would result in significantly increased background in the data. The sample was slowly cooled to $\sim 115 \mathrm{~K}$ during adsorption to allow for the sample to reach equilibrium without condensation of the methane. Following complete adsorption of the dose, as evidenced by a zero pressure reading inside the system, samples were cooled to $8 \mathrm{~K}$ for NPD measurements.

NPD patterns were analyzed using Rietveld analysis, ${ }^{78}$ as implemented in EXPGUI/GSAS. ${ }^{79,80}$ The starting model for the $\mathrm{CD}_{4}$-loaded materials was taken from our previously reported structures for all of the $\mathrm{M}_{2}$ (dobdc) analogues. ${ }^{81,82}$ Fourier difference methods were employed to locate the adsorbed $\mathrm{CD}_{4}$ molecules. ${ }^{83}$ During the refinements the $\mathrm{C}-\mathrm{D}$ and $\mathrm{D}-\mathrm{D}$ distances of the $\mathrm{CD}_{4}$ molecule were constrained to chemically reasonable values (bond distances that are consistent with average values from crystal structures in the Cambridge Structural Database with atoms in similar chemical environments). The fractional occupancy of all five atoms in the molecule was constrained to be identical, and the isotropic displacement parameter value of all four deuterium atoms was constrained to be identical. All refined atomic parameters for all structures are included in Section 5 of the Supporting Information, along with final Rietveld plots and selected Fourier difference maps.

\section{RESULTS AND DISCUSSION}

The performance of the eight vdW functionals and $\mathrm{PBE}$ in the $\mathrm{M}_{2}(\mathrm{dobdc})$ series $(\mathrm{M}=\mathrm{Mg}, \mathrm{Mn}, \mathrm{Fe}, \mathrm{Co}, \mathrm{Ni}, \mathrm{Cu}$, and $\mathrm{Zn})$ for the binding of three guests has been explored. The guests were chosen to represent a range of binding strengths, with $\mathrm{CH}_{4}$ binding the weakest, followed by $\mathrm{CO}_{2}$, and then $\mathrm{H}_{2} \mathrm{O}$. While other benchmarking studies have been performed for this family of materials, ${ }^{35,84}$ we present new NPD structural data and QMC calculations with which to compare the DFT results. Furthermore, we will explore whether the relative performance of the 
functionals varies depending on the metal or binding strength of the guest.

4.1. Comparison of DFT Results with Quantum Monte Carlo in $\mathrm{Fe}_{2}$ (dobdc). Although a significant amount of experimental data is available for the $\mathrm{M}_{2}$ (dobdc) series, electronic structure methods can be performed for systems independent of experiment, in addition to providing complementary data in collaborative studies. Furthermore, as has often been discussed in the literature, making comparisons between experiment and simulation for porous materials is not always straightforward. $^{3,5,20}$ Differences between experimental geometries and errors intrinsic in measurements can lead to indirect comparisons. Additionally, experimental measurements are of binding enthalpies, and calculating enthalpies requires including thermal effects. While outside the scope of this work, research into accurately computing the vibrational contributions to the enthalpy, in particular, anharmonic effects, is another active area of research, and the use of the harmonic approximation in this work may contribute to some of the differences between our calculations and experiment. ${ }^{85,86}$ Since this work is focused on how well different DFT functionals treat the electronic contributions to the enthalpy of adsorption, a higher-level quantum chemical method could be used to compute electronic binding energies that can be directly compared to DFT electronic binding energies. With this in mind, QMC calculations were performed for $\mathrm{CO}_{2}$ in $\mathrm{Fe}_{2}$ (dobdc) to determine the viability of this approach to determine the accuracy of the DFT binding energies. Since geometry optimizations for the system sizes being considered here are beyond reach with QMC, structures were taken from DFT, as is common practice in QMC-based determinations of binding energies (see Al-Hamdani et al. ${ }^{87}$ and Ma et al. ${ }^{88}$ ). So as to get a clean comparison with the DFT functionals, binding energy calculations were repeated for each functional at the same geometry used in the QMC calculations. The results obtained from these calculations are reported in Table 1. The QMC

Table 1. $\mathrm{CO}_{2}$-Framework Interaction Energies $(\mathrm{kJ} / \mathrm{mol})$ in $\mathrm{Fe}_{2}$ (dobdc) Computed ${ }^{a}$ at a Fixed Geometry with Several DFT Functionals and QMC

\begin{tabular}{llc}
\multicolumn{1}{c}{ functional } & $\begin{array}{c}\text { electronic binding energy } \\
\text { (fixed Lee et al. }\end{array}$ geometry) & $\begin{array}{c}\text { electronic binding energy } \\
\text { (opt. geometry, Section 4.3) }\end{array}$ \\
PBE & -13.6 & -13.1 \\
optB88-vdW & -45.4 & -46.5 \\
optB86-vdW & -45.2 & -46.1 \\
vdW-DF2 & -38.1 & -38.1 \\
rev-vdW-DF2 & -36.4 & -37.3 \\
PBE-D2 & -33.5 & -33.9 \\
PBE-D3 & -34.7 & -34.5 \\
PBE-D3 BJ & -35.3 & -35.3 \\
QMC & $-44.0(2.0)$ to $-41.0(2.0)$ &
\end{tabular}

${ }^{a}$ DFT binding energies where the position of $\mathrm{CO}_{2}$ has been optimized are also reported for comparison. The stochastic error bars on the QMC binding energies are given in parentheses, and the two values correspond to the binding energy obtained using the approach of Chiesa et al. $^{73}$ and the MPC interaction ${ }^{70}$ to correct for finite size errors, respectively.

estimate of the binding energy is ca. $40-45 \mathrm{~kJ} / \mathrm{mol}$; specifically, two values of $44.0 \pm 2.0$ and $41.0 \pm 2.0 \mathrm{~kJ} / \mathrm{mol}$ have been obtained depending on how finite size effects are taken in to account. In the former, finite size effects were corrected for with the method of Chiesa et al., ${ }^{73}$ and in the latter the model periodic Coulomb (MPC) interaction approach was used. ${ }^{70}$
Because of the size of the unit cell being considered and the enormous associated computational cost of the QMC simulations, explicit convergence tests in larger unit cells are beyond reach, and so we do not attempt to discriminate between the two values obtained.

Turning to the comparison between QMC and DFT, we find that $\mathrm{PBE}$ performs poorly and underestimates the binding energy by more than $20 \mathrm{~kJ} / \mathrm{mol}$. This is consistent with general understanding that standard GGAs such as PBE do not properly account for dispersion. ${ }^{15,17,21,22}$ All of the van der Waals inclusive functionals do significantly better. On the one hand, the DF functionals are $1-2 \mathrm{~kJ} / \mathrm{mol}$ below the lower end of the range predicted by QMC. On the other hand, the DF2 functionals fall on the other end of the QMC predicted range and bind $3-5 \mathrm{~kJ} / \mathrm{mol}$ weaker than the upper end of the QMC range. The $-D$ functionals also bind weaker than the upper end of the QMC range by $6-8 \mathrm{~kJ} / \mathrm{mol}$. In Table 1 , we also included results (taken from Section 4.3) where the position of $\mathrm{CO}_{2}$ was optimized with each functional. These data are included here to demonstrate the effect of using a fixed geometry in DFT with the goal of providing some indication of the potential effect of not optimizing with QMC. These calculations show that at the DFT level changes in structures lead to at most $\sim 1 \mathrm{~kJ} / \mathrm{mol}$ change in binding energy. The differences in binding energy between the respective are larger than this; therefore, we expect the use of the fixed geometry to have a limited impact on the interpretation of our results.

Before closing, it is essential to compare the QMC value to the experimentally determined result. From the work of Queen et al., ${ }^{43}$ a value of $-33.2 \mathrm{~kJ} / \mathrm{mol}$ has been obtained for $\mathrm{CO}_{2}$ in $\mathrm{Fe}_{2}$ (dobdc). Adjusting this experimental value by adding an estimated $3.616 \mathrm{~kJ} / \mathrm{mol}$ for finite temperature and zero-point energy (ZPE) effects leads to value of $-36.8 \mathrm{~kJ} / \mathrm{mol}$. This is quite close but nonetheless slightly (4 to $7 \mathrm{~kJ} / \mathrm{mol}$ ) lower than the QMC estimated value of ca. $40-45 \mathrm{~kJ} / \mathrm{mol}$. It is beyond the scope of the current study to resolve this discrepancy, but doing so would make interesting work for the future. On the QMC side, it will require a careful consideration of finite size effects, timestep errors, and potential sensitivity of the results to the nature of the trial wave function or the geometry; however, the thermal corrections to the energy computed with DFT use the harmonic approximation, and this could contribute to the differences as well.

The importance of the choice of reference can already been seen. On the one hand, if the experimental value is used as the reference, the DF functionals over bind by $\sim 8 \mathrm{~kJ} / \mathrm{mol}$, while the vdW-DF2 functional over binds by only $1.3 \mathrm{~kJ} / \mathrm{mol}$. On the other hand, the rev-vdW-DF2 functional under binds by only $0.4 \mathrm{~kJ} / \mathrm{mol}$, and the $-\mathrm{D}$ functionals are under binding by $1-3 \mathrm{~kJ} / \mathrm{mol}$ (as opposed to 6-8 when compared to QMC). We again wish to emphasize that care must be taken when assigning the "best" functional if comparing only against experiment or only against another computational method such as QMC.

4.2. The Effect of the vdW Treatment on the Framework Geometry. In Section 4.1, we explored the effect of changing the computational method while keeping the geometry fixed. In this section, we will study the effect changing the computational method has on the optimized geometry of the framework. In the previous section, we used the PBE geometry of Lee et al. ${ }^{33}$ They compared the calculated PBE lattice constants and metal-oxygen distances with neutron and X-ray diffraction measurements as well as with other calculated values previously reported in the literature. In the diffraction experiments, 
temperatures ranged from 4 to $368 \mathrm{~K}$ depending on the experiment. Although in several cases, the temperature is very low, note that the DFT calculations are performed at $0 \mathrm{~K}$, and thermal and zero-point effects can thus contribute to the differences between the measured and calculated values. Here we compare our results with the measurements made at the lowest temperature (see Table 2 and Table S1). ${ }^{24,26,27,31,76,89,90}$ Two important trends are

Table 2. Experimental and Calculated ${ }^{a}$ Lattice Constants and Metal-Oxygen Distances for $\mathbf{M g}_{2}$ (dobdc) with PBE, optB88-vdW, optB86b-vdW, vdW-DF2, rev-vdW-DF2, PBE-D2, PBE-D3, PBE-D3 BJ, and M06-L

\begin{tabular}{|c|c|c|c|c|}
\hline \multirow[b]{2}{*}{ method } & \multicolumn{2}{|c|}{ lattice constant $(\AA)$} & \multicolumn{2}{|c|}{$\begin{array}{c}\mathrm{Mg}-\mathrm{O} \text { distance } \\
(\AA)\end{array}$} \\
\hline & $a$ & $c$ & apical & basal \\
\hline $\exp (\mathrm{ND} \text { at } 20 \mathrm{~K})^{89}$ & $25.921(2)$ & $6.8625(8)$ & 2.08 & 2.01 \\
\hline $\mathrm{PBE}^{33}$ & 26.07 & 6.93 & 2.02 & 2.04 \\
\hline optB88-vdW & 26.03 & 6.91 & 2.03 & 2.04 \\
\hline optB86b-vdW & 26.01 & 6.90 & 2.02 & 2.03 \\
\hline vdW-DF2 & 26.21 & 6.95 & 2.04 & 2.05 \\
\hline rev-vdW-DF2 & 26.01 & 6.91 & 2.02 & 2.03 \\
\hline PBE-D2 & 26.01 & 6.88 & 2.01 & 2.03 \\
\hline PBE-D3 & 26.04 & 6.92 & 2.02 & 2.04 \\
\hline PBE-D3 BJ & 26.03 & 6.91 & 2.02 & 2.04 \\
\hline M06-L & 25.78 & 6.83 & 1.99 & 2.01 \\
\hline
\end{tabular}

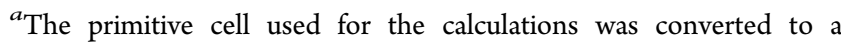
conventional cell to compare with experiment. The basal $\mathrm{Mg}-\mathrm{O}$ bond distances are the average of all four basal $\mathrm{Mg}-\mathrm{O}$ bonds.

considered here: the effect on the geometric parameters when the metal is changed and the performance of each functional compared to experiment (and one another). Note that the optimizations were performed using the rhombohedral unit cell, but they have been converted to the conventional trigonal cell for comparison with experimental results below.

The lattice parameters and $\mathrm{M}-\mathrm{O}$ bond distances change depending on the metal in the framework. In Figure 2, all of the functionals studied here can reproduce the general experimental ordering across the metals for both lattice constants and bond distances. The ordering in the lattice parameter $\mathbf{a}$ is $\mathrm{Mn}>\mathrm{Fe}>$ $\mathrm{Cu}>\mathrm{Mg}>\mathrm{Zn}>\mathrm{Co}>\mathrm{Ni}$, the ordering in the lattice parameter $\mathrm{c}$ is $\mathrm{Mn}>\mathrm{Mg}>\mathrm{Fe}>\mathrm{Zn}>\mathrm{Co}>\mathrm{Ni}>\mathrm{Cu}$, the ordering in the apical $\mathrm{M}-\mathrm{O}$ bond distance is $\mathrm{Cu}>\mathrm{Zn}>\mathrm{Mn}>\mathrm{Mg}>\mathrm{Fe}>\mathrm{Co}>\mathrm{Ni}$, and the ordering in the basal $\mathrm{M}-\mathrm{O}$ distance is $\mathrm{Zn}>\mathrm{Mn}>\mathrm{Fe}>\mathrm{Mg}>$ $\mathrm{Co}>\mathrm{Ni}>\mathrm{Cu}$. For cases where the differences between the experimental values is small, the ordering of two metals may deviate from those listed above for some functionals (i.e., the average basal $\mathrm{M}-\mathrm{O}$ distances for $\mathrm{Ni}$ and $\mathrm{Cu}$ are very close). If we take $\mathrm{Mg}_{2}$ (dobdc) as an example, the performance of the functionals for framework geometries can be observed. A complete list of geometric parameters is given in Table S1. The framework geometry optimized with the PBE functional has been compared to a larger set of experimental data by Lee et al. ${ }^{33}$ For the $\mathrm{Mg}$ framework with the experimental reference taken in this work by Liu et al., ${ }^{89}$ PBE deviates from experiment by $0.57 \%$ in lattice constant a and by $0.97 \%$ in lattice constant c. Aside from M06-L, all of the functionals yield larger lattice constants than experiment. On the one hand, for the functional with values closest to experiment (for $\mathrm{Mg}, \mathrm{PBE}-\mathrm{D} 2$ ), the a lattice constant has a percent deviation from experiment of $0.34 \%$, while the $\mathrm{c}$ constant deviates by $0.26 \%$. On the other hand, the vdW-DF2 functional deviates by $1.11 \%$ in a and $1.28 \%$ in $\mathrm{c}$ and is the farthest from experiment. M06-L has percent deviations of $0.54 \%$ in a and $0.47 \%$ in $c$, but the lattice constants are shorter than those of experiment. The remaining vdW functionals all do reasonably well and have deviations between 0.34 and $0.46 \%$ in a and $0.55-0.84 \%$ in $\mathbf{c}$.

If we use the percent deviation from experiment to characterize how well the functionals describe the $\mathrm{Mg}-\mathrm{O}_{\text {apical }}$ bond, we would determine that M06-L does the worst, as it deviates by $4.33 \%$, while vdW-DF2 does the best deviating by $1.92 \%$. This is a clear example of why we recommend taking care when making comparisons with one experimental measurement (in this case, a single distance from one experimental structure). The vdW-DF2 functional (one of the DF2 functionals) not only overestimates bond distances in our systems (Figure 2) but has been shown to do so in a variety of chemical systems. ${ }^{17}$ The fact that it is closest to experiment raises suspicion and is likely due to an elongated $\mathrm{Mg}-\mathrm{O}_{\text {apical }}$ distance in experiment. This could be due to an interaction at the metal center in experiment that effectively weakens the $\mathrm{M}-\mathrm{O}_{\text {apical }}$ bond. In fact, it is known that only $\sim 80 \%$ of the metal sites are accessible for small-molecule binding in the $\mathrm{Mg}_{2}$ (dobdc) structure. ${ }^{48}$

However, we can still use percent deviations as a guide if we take the PBE functional as a reference, since it has been previously shown to do reasonably well for metal-oxygen distances in this framework. On the one hand, M06-L predicts shorter bond distances and deviates from PBE by $1.5 \%$ for both the apical and basal distances. On the other hand, the PBE-D2, PBE-D3 BJ, optB86b-vdW, and rev-vdW-DF2 yield the same $\mathrm{M}-\mathrm{O}_{\text {apical }}$ distance as $\mathrm{PBE}$ and deviate by $0 \%, 0 \%, 0.5 \%$, and $0.5 \%$ in the average $\mathrm{M}-\mathrm{O}_{\text {basal }}$ distances, respectively. $\mathrm{PBE}-\mathrm{D} 2$ deviates by $0.5 \%$ in both the basal and apical distances, while optB88-vdW deviates by $0.5 \%$ in the apical distance but gives the same average value as $\mathrm{PBE}$ in the basal distances. Additionally, the vdW-DF2 functional deviates from PBE by $1.0 \%$ in the apical distance and $0.5 \%$ in the average basal distance. Since the vdW-DF2 distance has a smaller deviation from PBE than M06-L (but in opposite directions), it is possible that the "true" value is shorter than what is predicted by $\mathrm{PBE}$, since overall M06-L has been shown to yield better geometries than vdW-DF2. As a final note, although this discussion has focused on $\mathrm{Mg}$, the same analysis could be done for any of the metals. While some of the details in the discussion would change, the deviation between functionals is generally consistent regardless of the metal (i.e., we are not doing dramatically worse for one metal and better for another with a given functional). In particular, the overall conclusion is that, aside from vdW-DF2, the vdW corrected functionals do reasonably well at predicting geometries.

4.3. The Effect of the vdW Treatment on the Binding Energies and Guest Geometries. In this section, we explore the effect changing the computational method has on the binding energy and guest binding geometries. Since high-quality experimental data are a prerequisite for assessing the performance of the calculations in this section, before discussing our results we first introduce the structural data to which we will make comparisons. While NPD experiments had been reported for $\mathrm{CO}_{2}$ in the $\mathrm{M}_{2}$ (dobdc) series, ${ }^{43} \mathrm{CH}_{4}$ adsorption had not been measured. To characterize the coordination of the methane molecule at the metal site, NPD experiments were performed by dosing each of the $\mathrm{M}_{2}$ (dobdc) materials with $\mathrm{CD}_{4}$. An amount corresponding to $0.75 \mathrm{CD}_{4}$ molecules per metal atom was chosen to ensure that all of the gas would preferentially adsorb at the metal site without any adsorption at secondary sites. Fourier difference maps generated during the refinement process confirmed 

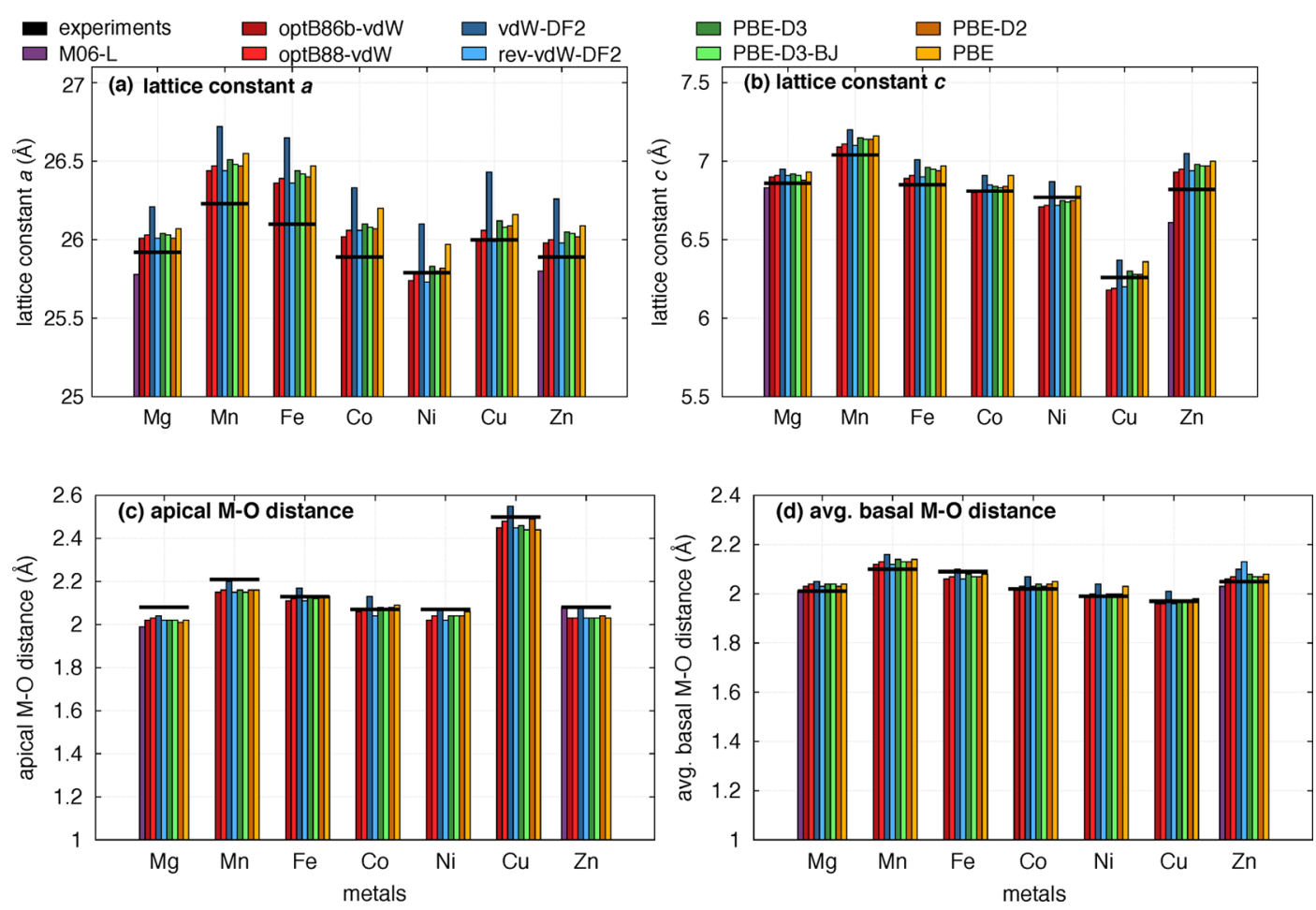

Figure 2. Calculated lattice constants and metal-oxygen distances for the $\mathrm{M}_{2}$ (dobdc) series with PBE, optB88-vdW, optB86b-vdW, vdW-DF2, rev-vdW-DF2, PBE-D2, PBE-D3, PBE-D3 BJ, and M06-L. The primitive cell used for the calculations was converted to a conventional cell to compare with experiment (Mg NPD at $20 \mathrm{~K},{ }^{89} \mathrm{Mn} \mathrm{XRD,},{ }^{31} \mathrm{Fe} N P D$ at $9 \mathrm{~K},{ }^{24} \mathrm{Co} \mathrm{XRD} \mathrm{at} 368 \mathrm{~K},{ }^{26} \mathrm{Ni}$ XRD at $295 \mathrm{~K},{ }^{27} \mathrm{Cu} \mathrm{XRD}$ at $100 \mathrm{~K},{ }^{76}$ and NPD at $10 \mathrm{~K}{ }^{43}$ ). The apical and basal $\mathrm{M}-\mathrm{O}$ distances are shown in Figure 1.

that all residual nuclear density was indeed located exclusively above the metal centers.

Figure 3 shows the local coordination of the $\mathrm{CD}_{4}$ molecule adsorbed at the metal site for each of the materials, and the interaction distances are listed in Table 3. For all of the materials, the $\mathrm{CD}_{4}$ molecule binds with a nearly identical geometry, where the metal interacts with three of the $\mathrm{D}$ atoms of the molecule, and the fourth is aligned nearly perpendicular to the basal plane. A slight tilting of the molecule is observed in the $\mathrm{Co}_{2}$ (dobdc) and $\mathrm{Ni}_{2}$ (dobdc) structures, where closer contacts with two of the $\mathrm{D}$ atoms occurs compared to the other materials. This tilting does not result in a closer metal-carbon distance, as the values for $\mathrm{Mg}_{2}$ (dobdc), $\mathrm{Mn}_{2}$ (dobdc), $\mathrm{Fe}_{2}$ (dobdc), $\mathrm{Co}_{2}$ (dobdc), and $\mathrm{Ni}_{2}$ (dobdc) all fall within 2.90 and $2.95 \AA$. Longer interaction distances are observed, as expected, for $\mathrm{Zn}_{2}$ (dobdc) and $\mathrm{Cu}_{2}$ (dobdc), where the metal-carbon distances increase to 3.09(1) and 3.28(1) Å, respectively. These observations correlate with long metal-gas interactions reported recently for $\mathrm{CO}$ and $\mathrm{CO}_{2}$ in $\mathrm{Zn}_{2}$ (dobdc) and $\mathrm{Cu}_{2}$ (dobdc) compared to the rest of the series, as well as with the DFT calculations presented herein. ${ }^{43,91}$

Along with the experimental structural data, experimental heats of adsorption are available for both $\mathrm{CO}_{2}$ and $\mathrm{CH}_{4}$. By comparing the measured and calculated binding energies (Figure 4a,b), we find that all of the functionals yield the correct metal dependence for guest binding. The trend in binding energies that is observed across the $\mathrm{M}_{2}$ (dobdc) series has been explained for $\mathrm{CO}_{2}$ in previous studies, such as those by Lee et al. and Poloni et al. ${ }^{33,84}$ The $\mathrm{CO}_{2}$ molecule bends slightly upon binding at the open-metal site thereby obtaining a dipole moment. Furthermore, the strength of binding between $\mathrm{CO}_{2}$ and the open metal center is not only due to a strong electrostatic interaction but also through the hybridization between the metal $\mathrm{d}_{z}{ }^{2}$ orbital and a
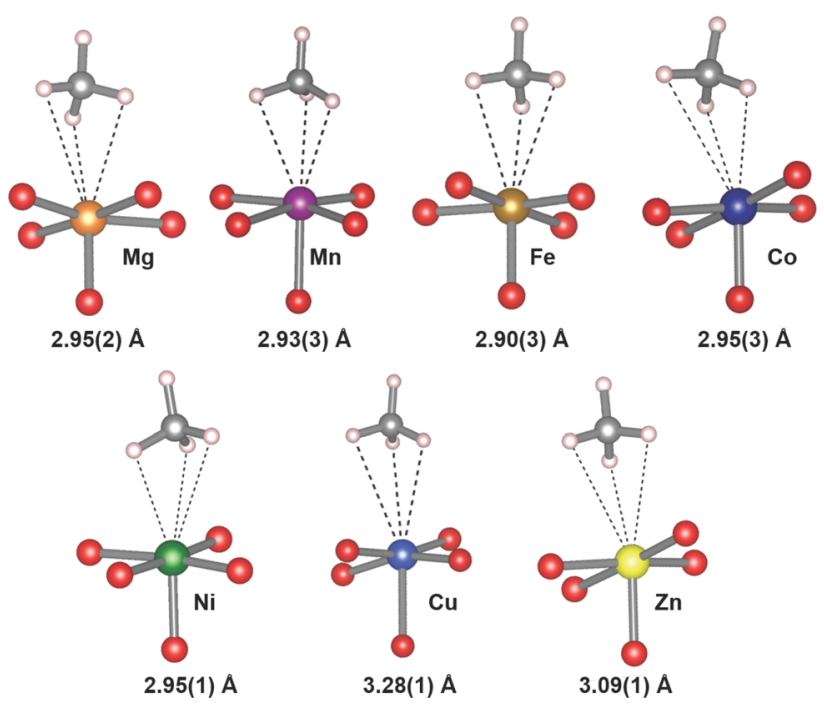

Figure 3. Methane molecule adsorbed at the metal site for the $\mathrm{M}_{2}$ (dobdc) series, as determined by NPD. The distance listed represents the distance from the respective metal center to the carbon atom of the $\mathrm{CD}_{4}$ molecule. Values in parentheses indicate one standard deviation.

lone pair on $\mathrm{CO}_{2}$. For $\mathrm{Mn}$ through $\mathrm{Co}$, the $\mathrm{d}_{z}{ }^{2}$ orbital is singly occupied; however, in $\mathrm{Cu}$ and $\mathrm{Zn}$ this orbital is doubly occupied, and the binding strength decreases. While no experimental data are available for $\mathrm{H}_{2} \mathrm{O}$ (Figure 4c), all of the functionals show the same metal dependence when compared to one another and following a trend similar to that of $\mathrm{CO}_{2}$. Additionally, since $\mathrm{CH}_{4}$ binds weaker and does not have a dipole, it is not surprising that the metal dependence is smaller with only $\mathrm{Cu}$ showing a significant difference in binding strength. 
Table 3. Metal-Carbon Distance and the Average of the Three Closest Metal-Deuterium Distances for the Methane Molecule Adsorbed at the Metal Site from the $\mathrm{M}_{2}(\mathrm{dobdc}) \cdot 1.5 \mathrm{CD}_{4}$ Structures Measured at $8 \mathrm{~K}$

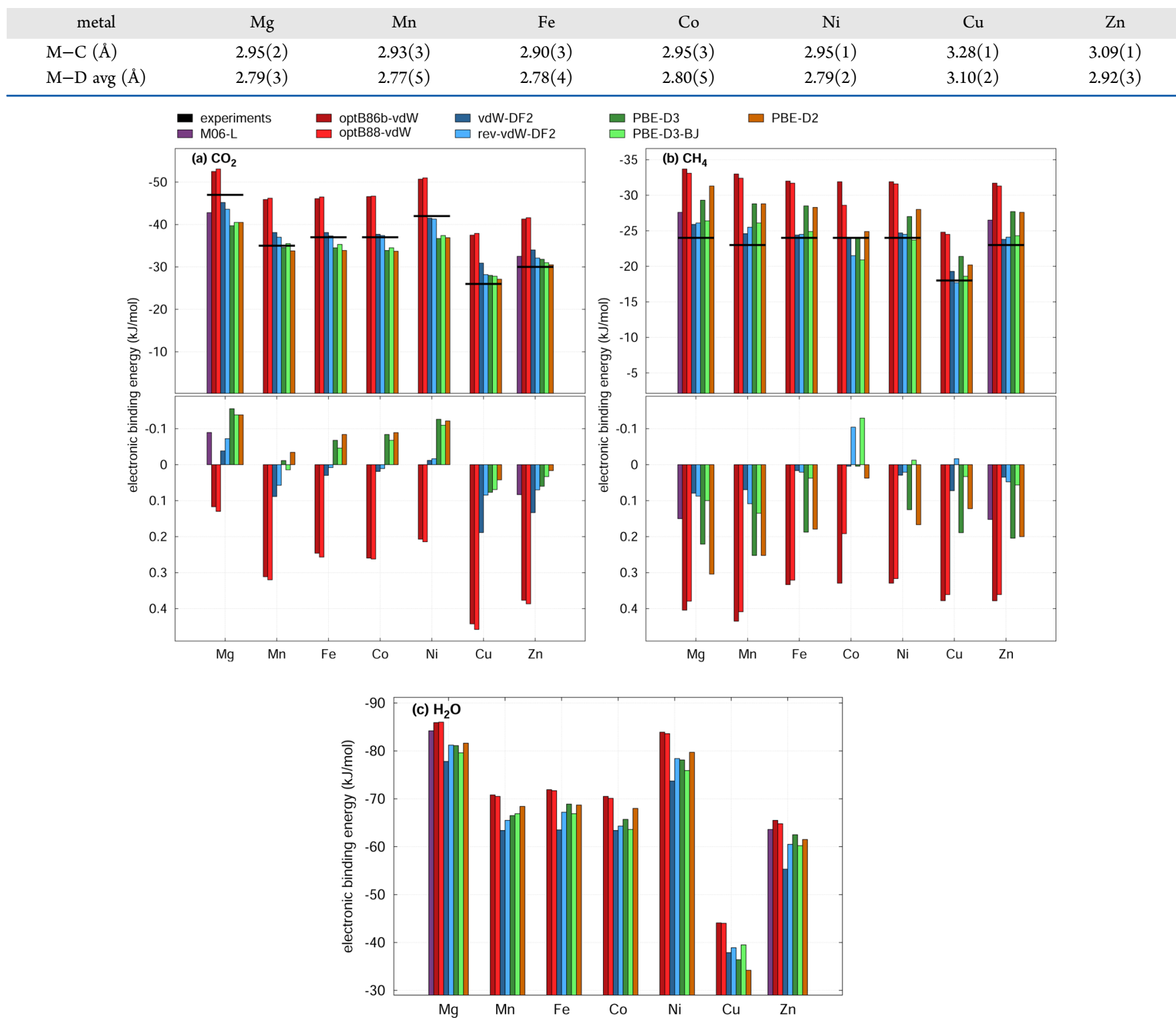

Figure 4. Electronic binding energies for eight functionals in the $\mathrm{M}_{2}$ (dobdc) series and available experimental data. To compare the experimental heat of adsorption ${ }^{43,81}$ with the calculated electronic binding energy, the harmonic vibrational corrections computed with the vdW-DF2 functional were subtracted from the experimental value for each case. The upper panels $(\mathrm{a}-\mathrm{c})$ show absolute values for $\mathrm{CO}_{2}, \mathrm{CH}_{4}$, and $\mathrm{H}_{2} \mathrm{O}$, respectively. The lower panels in $(a, b)$ indicate the relative differences to the experimental values.

The functionals were first compared with experimental binding energies (note that PBE was not included in this section, since the performance in Section 4.1 was so poor). The DF functionals over bind when compared to experiment (see Figure 4); however, binding energies improve for the DF2 functionals (see Section 4 of the Supporting Information for a discussion of an energy decomposition showing the differences arising from the vdW term). Furthermore, the $\mathrm{CO}_{2}$ binding energies for the two DF2 functionals differ at most by $2.7 \mathrm{~kJ} / \mathrm{mol}$ across the metal series, and the two DF functionals functionals differ at most by $0.6 \mathrm{~kJ} / \mathrm{mol}$. The two sets differ from one another by $\sim 9 \mathrm{~kJ} / \mathrm{mol}$. Although the difference in methane binding energies predicted between the two DF2 functionals remains small, rev-vdW-DF2 consistently predicts the binding energy to be $\sim 3 \mathrm{~kJ} / \mathrm{mol}$ weaker than vdW-DF2. This is also the case for the DF functionals, where
optB88-vdW binds only very slightly weaker than optB86bvdW for methane.

However, the $-\mathrm{D}$ functionals without Becke-Johnson (BJ) damping under bind for $\mathrm{CO}_{2}$ and over bind for methane when compared with experiment. For $\mathrm{CO}_{2}$ and $\mathrm{H}_{2} \mathrm{O}$, their performance is more similar to the DF2 functionals, while for methane their performance is between that of the DF and DF2 functionals. PBE-D3 $\mathrm{BJ}$ exhibits results very similar to the other $-\mathrm{D}$ and $\mathrm{DF} 2$ functionals for $\mathrm{CO}_{2}$ and $\mathrm{H}_{2} \mathrm{O}$; however, it remains close to the DF2 functionals for methane, while the other $-\mathrm{D}$ functionals bind stronger.

Finally, the M06-L binding energies for $\mathrm{CO}_{2}$ and $\mathrm{CH}_{4}$ are in reasonably good agreement with experiment but bind slightly stronger than the DF2 functionals. While this functional performed well across the board, the M06-L calculations required significantly more wall time than the other functionals tested herein, and SCF convergence was challenging. 

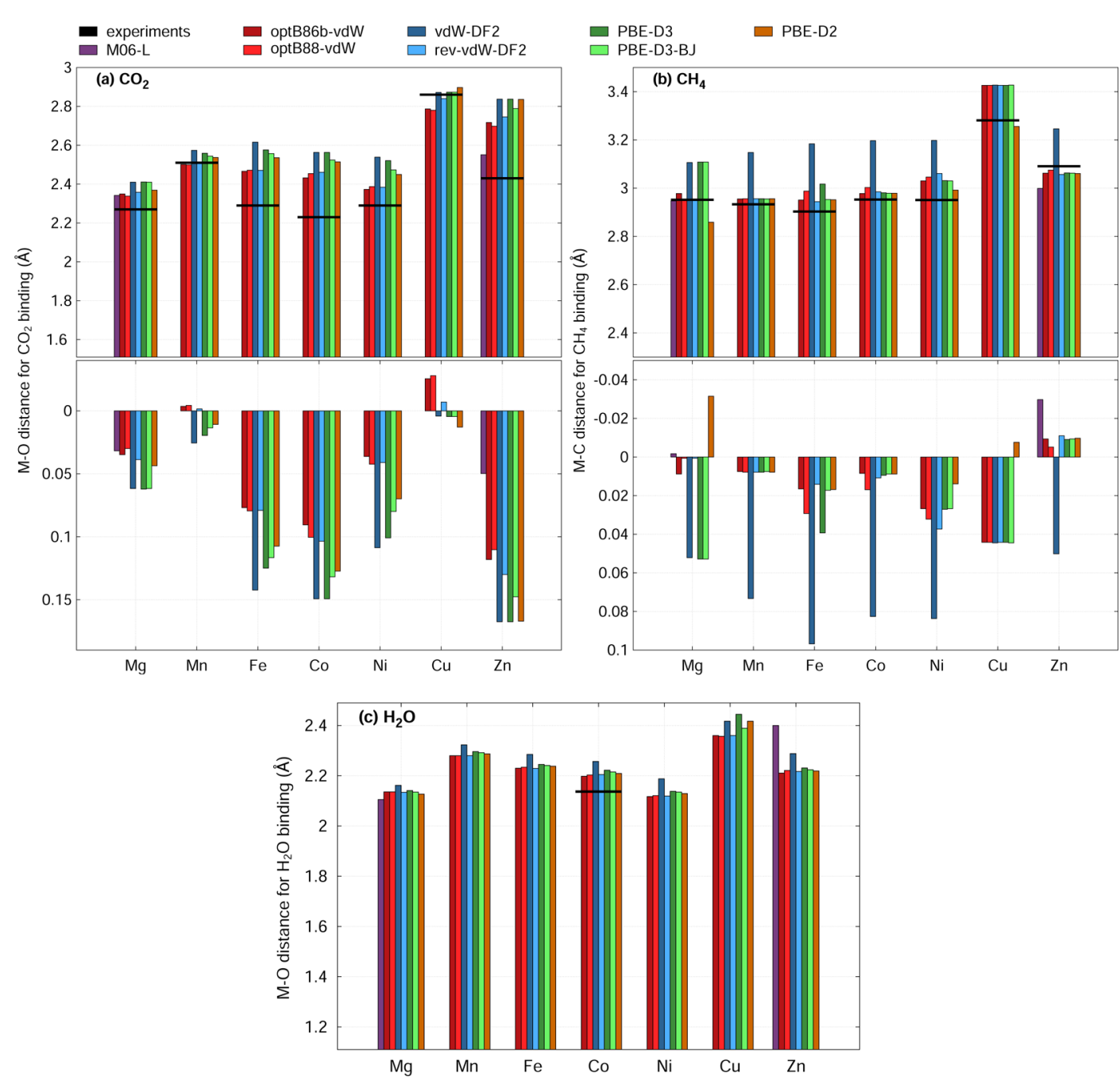

Figure 5. Guest binding distances (in $\AA$ ) for the eight functionals in the $\mathrm{M}_{2}$ (dobdc) series and experimental values when available. Experimental results for $\mathrm{CO}_{2}$ are from Queen et al., ${ }^{43}$ while those for $\mathrm{CH}_{4}$ are from this work, and those for $\mathrm{H}_{2} \mathrm{O}$ are from Mercado et al. ${ }^{92}$ The upper panels in $(\mathrm{a}-\mathrm{c})$ show absolute values for $\mathrm{CO}_{2}, \mathrm{CH}_{4}$, and $\mathrm{H}_{2} \mathrm{O}$, respectively. The lower panels in $(\mathrm{a}, \mathrm{b})$ indicate the relative differences to the experimental values.

The final measured and calculated property to be compared is the binding geometry of the guests. The selected parameters used to describe guest binding are the distances $\mathrm{M}-\mathrm{O}_{\mathrm{CO} 2}, \mathrm{M}-\mathrm{C}_{\mathrm{CH} 4}$, and $\mathrm{M}-\mathrm{O}_{\mathrm{H} 2 \mathrm{O}}$ (Figure 5). Although we did not have experimental heats of adsorption for $\mathrm{H}_{2} \mathrm{O}$, the $\mathrm{M}-\mathrm{O}_{\mathrm{H} 2 \mathrm{O}}$ distance was previously reported for $\mathrm{Co}_{2}(\mathrm{dobdc})$ at $100 \mathrm{~K}$ using single-crystal $\mathrm{X}$-ray diffraction (XRD). ${ }^{92}$ The experimental distance of 2.137(0) A is included as well.

As was the case with the lattice parameters, the DF functionals, rev-vdW-DF2, and M06-L have guest binding distances in better agreement with experimental data from NPD than the vdWDF2 functional and, in this case, to some extent the empirically corrected ones (Figure 5). We again take $\mathrm{Mg}_{2}$ (dobdc) as an example to demonstrate this point (Figure 5a,b). The experimental distances for guest binding are $2.270 \AA$ for $\mathrm{CO}_{2}$ and $2.952 \AA$ for $\mathrm{CH}_{4}$. On the one hand, the functionals that perform well (the DF functionals, rev-vdW-DF2, and M06-L) have percent deviations from experiment between 3.00 and $3.88 \%$ for $\mathrm{CO}_{2}$ and $0.07-0.88 \%$ for $\mathrm{CH}_{4}$. On the other hand, the vdW-DF2 functional results in the longest bond distances between metal and guest for all of the studied cases with percent deviations from experiment of $6.17 \%$ and $5.22 \%$ for $\mathrm{CO}_{2}$ and $\mathrm{CH}_{4}$, respectively. For the $-\mathrm{D}$ functionals, PBE-D3 and PBE-D3 BJ have percent deviations of $\sim 6 \%$ for $\mathrm{CO}_{2}$ and $\sim 5 \%$ for $\mathrm{CH}_{4}$, while PBE-D2 has slightly smaller deviations of $4.36 \%$ and $3.14 \%$ for $\mathrm{CO}_{2}$ and $\mathrm{CH}_{4}$, respectively. However, as was the case for the binding energies, in Figure 5 we see that the PBE-D2 functional is not consistently the best of the $-\mathrm{D}$ functionals (or put another way, the PBE-D3 functionals do not always have long distances like vdW-DF2). Given that the metal-oxygen distances for $\mathrm{CO}_{2}$ binding are overestimated in general with $\mathrm{Zn}$ having the largest differences from experiment, we again caution in reading too much into smaller differences between the experimental and calculated geometry for one particular framework. When the difference from experiment is plotted in Figure 5, the most dramatic deviations are for $\mathrm{CH}_{4}$, and it is once more that vdW-DF2 consistently has larger errors than the remainder of the functionals.

While very limited experimental data are available for $\mathrm{H}_{2} \mathrm{O}$ binding, recall that $\mathrm{H}_{2} \mathrm{O}$ binds stronger than $\mathrm{CO}_{2}$, and consequently, upon $\mathrm{H}_{2} \mathrm{O}$ adsorption, the bond between the apical oxygen atom and the metal center is elongated (Figure 5c). Thus, the strong interaction with $\mathrm{H}_{2} \mathrm{O}$ results in a weakening of the bond between the metal and the oxygen atom trans to the $\mathrm{H}_{2} \mathrm{O}$ within the framework. Additionally, the single-crystal diffraction experiment was performed for a loading higher than one $\mathrm{H}_{2} \mathrm{O}$ per metal center; therefore, in the experiment the $\mathrm{H}_{2} \mathrm{O}$ interacting to 
the metal is also interacting with neighboring $\mathrm{H}_{2} \mathrm{O}$ molecules and could contribute to differences between the measured and calculated values. Nevertheless, for $\mathrm{H}_{2} \mathrm{O}$ in $\mathrm{Co}_{2}$ (dobdc) vdW-DF2 again has the largest deviation from experiment.

\section{CONCLUSIONS}

While it is clear that small-molecule adsorption in MOFs cannot be studied without properly accounting for vdW interactions, multiple functionals are often not applied to systems in the literature. Fortunately, we find that the trends across metals in our system are reproduced with all eight vdW functionals; however, the assignment of a "best" functional is not straightforward.

If we compare with QMC calculations (Section 4.1), a clean comparison of electronic binding energies on identical structures can be made with DFT. These calculations were primarily performed to demonstrate that QMC, despite its enormous computational cost, is now feasible on systems as complex as MOFs thanks to recent developments. ${ }^{66}$ For the specific system examined, the QMC results suggest that the DF functionals bound $\mathrm{CO}_{2}$ by an energy between 1 and $2 \mathrm{~kJ} / \mathrm{mol}$ stronger than the lower end of the QMC range, while the DF2 functionals bound $\mathrm{CO}_{2} \sim 3-5 \mathrm{~kJ} / \mathrm{mol}$ less than then upper end of the range. The $-\mathrm{D}$ functionals underestimated binding when compared with QMC but would be considered better performing if compared to experiment. In short, if comparisons were made against QMC, all of the DF and DF2 functionals yield results just outside the error bars of the higher-level calculation. In the future, it will be interesting to perform more extensive comparisons between QMC, DFT, and well-defined experimental measurements of binding energies for more than one structure.

In Section 4.2 and 4.3 calculated geometric parameters and binding energies were compared to experiment. While additional factors aside from the treatment of dispersion contribute to deviations between measured and calculated values making this comparison indirect, comparison with experiment is nevertheless important, and making meaningful comparisons with experiment requires reliable experimental measurements. For this reason, NPD measurements were performed for $\mathrm{CD}_{4}$ in the $\mathrm{M}_{2}$ (dobdc) series. These data were not previously available, and they have been reported here for the first time. Both calculated guest binding distances and framework geometric parameters are systematically overestimated with the vdW-DF2 functional, but the newest of the DF2 functionals, rev-vdW-DF2, corrects for the overestimation of bond distances while retaining good binding energies; therefore, we recommend using this functional over vdW-DF2. The remaining vdW functionals also do well for predicting framework and guest binding geometries. Comparisons with experimental heat of adsorption measurements suggest that the DF functionals systematically overestimate the binding energy, while the DF2 functionals correct for this and provide a good description of binding energies. The $-\mathrm{D}$ functionals perform reasonably well, in some cases on par with the DF2 functionals, but in other cases over bind (with energies falling between the DF and DF2 functionals). While M06-L was only studied for the $\mathrm{Mg}$ and $\mathrm{Zn}$ frameworks and convergence with this functional can be challenging, it performs well for both binding energies and geometries.

\section{ASSOCIATED CONTENT}

\section{S Supporting Information}

The Supporting Information is available free of charge on the ACS Publications website at DOI: 10.1021/acs.jpca.7b00076.
The coordinates for DFT-optimized geometries, values of the data plotted in the main text, additional data from DFT convergence tests, and crystal structure details and Rietveld refinement plots (PDF)

$\mathrm{X}$-ray crystallographic information, additional data (ZIP)

\section{AUTHOR INFORMATION}

\section{ORCID 중}

Bess Vlaisavljevich: 0000-0001-6065-0732

Angelos Michaelides: 0000-0002-9169-169X

Berend Smit: 0000-0003-4653-8562

\section{Notes}

The authors declare no competing financial interest.

\section{ACKNOWLEDGMENTS}

B.V. was supported by the Center for Gas Separations Relevant to Clean Energy Technologies, an Energy Frontier Research Center, funded by the U.S. Department of Energy (DOE), Office of Science, Office of Basic Energy Sciences, under Award No. DE-SC0001015. J.M.H. was supported by the Deutsche Forschungsgemeinschaft (DFG, priority program SPP 1570). A.M. and B.S. have received funding from the European Research Council under the European Union's Seventh Framework Programme (FP/2007-2013)/ERC Grant No. 616121 (HeteroIce project) and under the European Union's Horizon 2020 research and innovation programme (Grant No. 666983, MaGic), respectively, and A.M. received funding from the Royal Society through a Wolfson Research Merit Award. Portions of this work were supported by the Molecular Foundry, funded by the U.S. DOE, Office of Science, Office of Basic Energy Sciences. This research used resources as part of an INCITE project (awarded to D.A.) at the Oak Ridge National Laboratory (Titan), which is supported by the Office of Science of the U.S. DOE under Contract No. DEAC05-00OR22725. This research also used resources of the Argonne Leadership Computing Facility at Argonne National Laboratory, which is supported by the Office of Science of the U.S. DOE under Contract No. DE-AC02-06CH11357 and National Energy Research Scientific Computing Center, a DOE Office of Science User Facility supported by the Office of Science of the U.S. DOE under Contract No. DE-AC02-05CH11231.

\section{REFERENCES}

(1) Furukawa, H.; Cordova, K. E.; O’Keeffe, M.; Yaghi, O. M. The Chemistry and Applications of Metal-Organic Frameworks. Science 2013, 341, 1230444.

(2) D’Alessandro, D. M.; Smit, B.; Long, J. R. Carbon Dioxide Capture: Prospects for New Materials. Angew. Chem., Int. Ed. 2010, 49, 60586082.

(3) Lee, J. S.; Vlaisavljevich, B.; Britt, D. K.; Brown, C. M.; Haranczyk, M.; Neaton, J. B.; Smit, B.; Long, J. R.; Queen, W. L. Understanding Small-Molecule Interactions in Metal-Organic Frameworks: Coupling Experiment with Theory. Adv. Mater. 2015, 27, 5785-5796.

(4) Evans, J. D.; Fraux, G.; Gaillac, R.; Kohen, D.; Trousselet, F.; Vanson, J.-M.; Coudert, F.-X. Computational Chemistry Methods for Nanoporous Materials. Chem. Mater. 2017, 29, 199-212.

(5) Odoh, S. O.; Cramer, C. J.; Truhlar, D. G.; Gagliardi, L. QuantumChemical Characterization of the Properties and Reactivities of MetalOrganic Frameworks. Chem. Rev. 2015, 115, 6051-6111.

(6) Keskin, S. Recent Advances in Molecular Dynamics Simulations of Gas Diffusion in Metal Organic Frameworks. In Molecular DynamicsTheoretical Developments and Applications in Nanotechnology; Wang, L., Ed.; InTech, 2012; pp 225-280. 
(7) Salles, F.; Ghoufi, A.; Maurin, G.; Bell, R. G.; Mellot-Draznieks, C.; Férey, G. Molecular Dynamics Simulations of Breathing MOFs: Structural Transformations of MIL-53(Cr) upon Thermal Activation and CO2 Adsorption. Angew. Chem., Int. Ed. 2008, 47, 8487-8491.

(8) Coupry, D. E.; Addicoat, M. A.; Heine, T. Extension of the Universal Force Field for Metal-Organic Frameworks. J. Chem. Theory Comput. 2016, 12, 5215-5225.

(9) Llewellyn, P. L.; Maurin, G.; Devic, T.; Loera-Serna, S.; Rosenbach, N.; Serre, C.; Bourrelly, S.; Horcajada, P.; Filinchuk, Y.; Férey, G. Prediction of the Conditions for Breathing of Metal Organic Framework Materials Using a Combination of X-ray Powder Diffraction, Microcalorimetry, and Molecular Simulation. J. Am. Chem. Soc. 2008, 130, 12808-12814.

(10) Mellot-Draznieks, C.; Serre, C.; Surblé, S.; Audebrand, N.; Férey, G. Very Large Swelling in Hybrid Frameworks: A Combined Computational and Powder Diffraction Study. J. Am. Chem. Soc. 2005, 127, 16273-16278.

(11) Jawahery, S.; Simon, C. M.; Braun, E.; Witman, M.; Tiana, D.; Vlaisavljevich, B.; Smit, B. Adsorbate-Induced Lattice Deformation in IRMOF-74 Series. Nat. Commun. 2017, 8, 13945.

(12) Kümmel, S.; Kronik, L. Orbital-Dependent Density Functionals: Theory and Applications. Rev. Mod. Phys. 2008, 80, 3-60.

(13) Johnson, E. R; Mackie, I. D.; DiLabio, G. A. Dispersion Interactions in Density Functional Theory. J. Phys. Org. Chem. 2009, 22, 1127-1135.

(14) Tkatchenko, A.; Romaner, L.; Hofmann, O. T.; Zojer, E.; Ambrosch-Draxl, C.; Scheffler, M. Van der Waals Interactions Between Organic Adsorbates and at Organic/Inorganic Interfaces. MRS Bull. 2010, 35, 435-442.

(15) Grimme, S. Density Functional Theory with London Dispersion Corrections. Wiley Interdiscip. Rev. Comput. Mol. Sci. 2011, 1, 211-228.

(16) Klimeš, J.; Michaelides, A. Perspective: Advances and Challenges in Treating Van der Waals Dispersion Forces in Density Functional Theory. J. Chem. Phys. 2012, 137, 120901.

(17) Berland, K.; Cooper, V. R.; Lee, K.; Schröder, E.; Thonhauser, T.; Hyldgaard, P.; Lundqvist, B. I. Van der Waals Forces in Density Functional Theory. Phys. Rev. B 2014, 89, 035412.

(18) Dion, M.; Rydberg, H.; Schröder, E.; Langreth, D. C.; Lundqvist, B. I. Van der Waals Density Functional for General Geometries. Phys. Rev. Lett. 2004, 92, 246401.

(19) Lee, K.; Murray, É. D.; Kong, L.; Lundqvist, B. I.; Langreth, D. C. Higher-Accuracy Van der Waals Density Functional. Phys. Rev. B: Condens. Matter Mater. Phys. 2010, 82, 81101.

(20) Yang, Q.; Liu, D.; Zhong, C.; Li, J. R. Development of Computational Methodologies for Metal-Organic Frameworks and their Application in Gas Separations. Chem. Rev. 2013, 113, 8261-323.

(21) Grimme, S.; Hansen, A.; Brandenburg, J. G.; Bannwarth, C. Dispersion-Corrected Mean-Field Electronic Structure Methods. Chem. Rev. 2016, 116, 5105-5154.

(22) Hermann, J.; DiStasio, R. A.; Tkatchenko, A. First-Principles Models for van der Waals Interactions in Molecules and Materials: Concepts, Theory, and Applications. Chem. Rev. 2017, 117, 4714-4758.

(23) Witte, J.; Mardirossian, N.; Neaton, J. B.; Head-Gordon, M. Assessing DFT-D3 Damping Functions Across Widely Used Density Functionals: Can We Do Better? J. Chem. Theory Comput. 2017, ASAP.10.1021/acs.jctc.7b00176

(24) Bloch, E. D.; Murray, L. J.; Queen, W. L.; Chavan, S.; Maximoff, S. N.; Bigi, J. P.; Krishna, R.; Peterson, V. K.; Grandjean, F.; Long, G. J.; Smit, B.; Bordiga, S.; Brown, C. M.; Long, J. R. Selective Binding of O2 Over N2 in a Redox-Active Metal-Organic Framework with Open Iron(II) Coordination Sites. J. Am. Chem. Soc. 2011, 133, 14814-14822.

(25) Rosi, N. L.; Kim, J.; Eddaoudi, M.; Chen, B.; O’Keeffe, M.; Yaghi, O. M. Rod Packings and Metal-Organic Frameworks Constructed from Rod-Shaped Secondary Building Units. J. Am. Chem. Soc. 2005, 127, $1504-1518$.

(26) Dietzel, P. D.; Morita, Y.; Blom, R.; Fjellvag, H. An In Situ HighTemperature Single-Crystal Investigation of a Dehydrated MetalOrganic Framework Compound and Field-Induced Magnetization of
One-Dimensional Metal-Oxygen Chains. Angew. Chem., Int. Ed. 2005, 44, 6354-6358

(27) Dietzel, P. D.; Panella, B.; Hirscher, M.; Blom, R.; Fjellvag, H. Hydrogen Adsorption in a Nickel Based Coordination Polymer with Open Metal Sites in the Cylindrical Cavities of the Desolvated Framework. Chem. Commun. 2006, 959-961.

(28) Dietzel, P. D. C.; Blom, R; Fjellvåg, H. Base-Induced Formation of Two Magnesium Metal-Organic Framework Compounds with a Bifunctional Tetratopic Ligand. Eur. J. Inorg. Chem. 2008, 2008, 36243632.

(29) Dietzel, P. D. C.; Johnsen, R. E.; Blom, R.; Fjellvåg, H. Structural Changes and Coordinatively Unsaturated Metal Atoms on Dehydration of Honeycomb Analogous Microporous Metal-Organic Frameworks. Chem. - Eur. J. 2008, 14, 2389-2397.

(30) Caskey, S. R.; Wong-Foy, A. G.; Matzger, A. J. Dramatic Tuning of Carbon Dioxide Uptake via Metal Substitution in a Coordination Polymer with Cylindrical Pores. J. Am. Chem. Soc. 2008, 130, 1087010871.

(31) Zhou, W.; Yildirim, T.; Wu, H.; et al. Enhanced H2 Adsorption in Isostructural Metal-Organic Frameworks with Open Metal Sites: Strong Dependence. J. Am. Chem. Soc. 2008, 130, 15268-15269.

(32) Bhattacharjee, S.; Choi, J. S.; Yang, S. T.; Choi, S. B.; Kim, J.; Ahn, W. S. Solvothermnal Synthesis of Fe-MOF-74 and its Catalytic Properties in Phenol Hydroxylation. J. Nanosci. Nanotechnol. 2010, 10, 135-141.

(33) Lee, K.; Howe, J. D.; Lin, L.-C.; Smit, B.; Neaton, J. B. Small Molecule Adsorption in Open-Site Metal-Organic Frameworks: A Systematic Density Functional Theory Study for Rational Design. Chem. Mater. 2015, 27, 668-678.

(34) Canepa, P.; Arter, C. A.; Conwill, E. M.; Johnson, D. H.; Shoemaker, B. A.; Soliman, K. Z.; Thonhauser, T. High-Throughput Screening of Small-Molecule Adsorption in MOF. J. Mater. Chem. A 2013, 1, 13597-13604.

(35) Rana, M. K.; Koh, H. S.; Zuberi, H.; Siegel, D. J. Methane Storage in Metal-Substituted Metal-Organic Frameworks: Thermodynamics, Usable Capacity, and the Impact of Enhanced Binding Sites. J. Phys. Chem. C 2014, 118, 2929-2942.

(36) Sillar, K.; Sauer, J. Ab Initio Prediction of Adsorption Isotherms for Small molecules in Metal-Organic Frameworks: the Effect of Lateral Interactions for Methane/CPO-27-Mg. J. Am. Chem. Soc. 2012, 134, 18354-18365.

(37) Poloni, R.; Smit, B.; Neaton, J. B. CO2 Capture by MetalOrganic Frameworks with Van der Waals Density Functionals. J. Phys. Chem. A 2012, 116, 4957-4964.

(38) Wu, H.; Simmons, J. M.; Srinivas, G.; Zhou, W.; Yildirim, T. Adsorption Sites and Binding Nature of $\mathrm{CO} 2$ Capture in Prototypical Metal-Organic Frameworks: A Combined Neutron Diffraction and First-Principles Study. J. Phys. Chem. Lett. 2010, 1, 1946-1951.

(39) Park, J.; Kim, H.; Han, S. S.; Jung, Y. Tuning Metal-Organic Frameworks with Open-Metal Sites and Its Origin for Enhancing CO2 Affinity by Metal Substitution. J. Phys. Chem. Lett. 2012, 3, 826-829.

(40) Yu, D. 1.; Yazaydin, A. O.; Lane, J. R.; Dietzel, P. D. C.; Snurr, R. Q. A Combined Experimental and Quantum Chemical Study of CO2 Adsorption in the Metal-Organic Framework CPO-27 with Different Metals. Chem. Sci. 2013, 4, 3544-3566.

(41) Rana, M. K.; Koh, H. S.; Hwang, J.; Siegel, D. J. Comparing Van der Waals Density Functionals for CO2 Adsorption in Metal Organic Frameworks. J. Phys. Chem. C 2012, 116, 16957-16968.

(42) Koh, H. S.; Rana, M. K.; Hwang, J.; Siegel, D. J. Thermodynamic Screening of Metal-Substituted MOFs for Carbon Capture. Phys. Chem. Chem. Phys. 2013, 15, 4573-4581.

(43) Queen, W. L.; Hudson, M.; Bloch, E.; Mason, J. A.; Gonzalez, M.; Lee, J.; Gygi, D.; Howe, J.; Lee, K.; Darwish, T. A.; James, M.; Peterson, V. K.; Teat, S. J.; Smit, B.; Neaton, J. B.; Long, J. R.; Brown, C. B. Comprehensive Study of Carbon Dioxide Adsorption in the MetalOrganic Frameworks M2(dobdc) $(\mathrm{M}=\mathrm{Mg}, \mathrm{Mn}, \mathrm{Fe}, \mathrm{Co}, \mathrm{Ni}, \mathrm{Cu}, \mathrm{Zn})$. Chem. Sci. 2014, 5, 4569-4581.

(44) Valenzano, L.; Civalleri, B.; Chavan, S.; Palomino, G. T.; Arean, C. O.; Bordiga, S. Computational and Experimental Studies on the 
Adsorption of CO, N2, and CO2 on Mg-MOF-74. J. Phys. Chem. C 2010, 114, 11185-11191.

(45) Valenzano, L.; Civalleri, B.; Sillar, K.; Sauer, J. Heats of Adsorption of $\mathrm{CO}$ and $\mathrm{CO} 2$ in Metal-Organic Frameworks: Quantum Mechanical Study of CPO-27-M ( $\mathrm{M}=\mathrm{Mg}, \mathrm{Ni}, \mathrm{Zn})$. J. Phys. Chem. C 2011, 115, 21777-21784.

(46) Hou, X.-J.; He, P.; Li, H.; Wang, X. Understanding the Adsorption Mechanism of $\mathrm{C} 2 \mathrm{H} 2, \mathrm{CO} 2$, and $\mathrm{CH} 4$ in Isostructural Metal-Organic Frameworks with Coordinately Unsaturated Metal Sites. J. Phys. Chem. C 2013, 117, 2824-2834.

(47) Verma, P.; Xu, X.; Truhlar, D. G. Adsorption on Fe-MOF-74 for C1-C3 Hydrocarbon Separation. J. Phys. Chem. C 2013, 117, 1264812660 .

(48) Dzubak, A. L.; Lin, L. C.; Kim, J.; Swisher, J. A.; Poloni, R.; Maximoff, S. N.; Smit, B.; Gagliardi, L. Ab Initio Carbon Capture in Open-Site Metal-Organic Frameworks. Nat. Chem. 2012, 4, 810-816.

(49) Borycz, J.; Lin, L.-C.; Bloch, E. D.; Kim, J.; Dzubak, A. L.; Maurice, R.; Semrouni, D.; Lee, K.; Smit, B.; Gagliardi, L. CO2 Adsorption in Fe2(dobdc): A Classical Force Field Parameterized from Quantum Mechanical Calculations. J. Phys. Chem. C 2014, 118, 12230-12240.

(50) Valvekens, P.; Vandichel, M.; Waroquier, M.; Van Speybroeck, V.; De Vos, D. Metal-dioxidoterephthalate MOFs of the MOF-74 type: Microporous basic catalysts with well-defined active sites. J. Catal. 2014, $317,1-10$.

(51) Palomino Cabello, C.; Gómez-Pozuelo, G.; Opanasenko, M.; Nachtigall, P.; Čejka, J. Metal-Organic Frameworks M-MOF-74 and M-MIL-100: Comparison of Textural, Acidic, and Catalytic Properties. ChemPlusChem 2016, 81, 828-835.

(52) Perdew, J. P.; Burke, K.; Ernzerhof, M. Generalized Gradient Approximation Made Simple. Phys. Rev. Lett. 1996, 77, 3865-3868.

(53) Klimeš, J.; Bowler, D. R.; Michaelides, A. Chemical Accuracy for the Van der Waals Density Functional. J. Phys.: Condens. Matter 2010, 22, 022201.

(54) Klimeš, J.; Bowler, D. R.; Michaelides, A. Van der Waals Density Functionals Applied to Solids. Phys. Rev. B: Condens. Matter Mater. Phys. 2011, 83, 195131.

(55) Hamada, I. Van der Waals Density Functional Made Accurate. Phys. Rev. B: Condens. Matter Mater. Phys. 2014, 89, 121103.

(56) Grimme, S. Semiempirical GGA-Type Density Functional Constructed with a Long-Range Dispersion Correction. J. Comput. Chem. 2006, 27, 1787-1799.

(57) Grimme, S.; Antony, J.; Ehrlich, S.; Krieg, H. A Consistent and Accurate $\mathrm{Ab}$ Initio Parametrization of Density Functional Dispersion Correction (DFT-D) for the 94 Elements H-Pu. J. Chem. Phys. 2010, 132, 154104.

(58) Grimme, S.; Ehrlich, S.; Goerigk, L. Effect of the Damping Function in Dispersion Corrected Density Functional Theory. J. Comput. Chem. 2011, 32, 1456-1465.

(59) Zhao, Y.; Truhlar, D. G. A New Local Density Functional for Main-Group Thermochemistry, Transition Metal Bonding, Thermochemical Kinetics, and Noncovalent Interactions. J. Chem. Phys. 2006, $125,194101$.

(60) Zhao, Y.; Truhlar, D. G. Density Functionals with Broad Applicability in Chemistry. Acc. Chem. Res. 2008, 41, 157-167.

(61) Kresse, G.; Joubert, D. From Ultrasoft Pseudopotentials to the Projector Augmented-Wave Method. Phys. Rev. B: Condens. Matter Mater. Phys. 1999, 59, 1758-1775.

(62) Dudarev, S. L.; Savrasov, S. Y.; Humphreys, C. J.; Sutton, A. P.; et al. Electron-Energy-Loss Spectra and the Structural Stability of Nickel Oxide: An LSDA+U Study. Phys. Rev. B: Condens. Matter Mater. Phys. 1998, 57, 1505-1509.

(63) Mann, G. W.; Lee, K.; Cococcioni, M.; Smit, B.; Neaton, J. B. First-Principles Hubbard U Approach for Small Molecule Binding in Metal-Organic Frameworks. J. Chem. Phys. 2016, 144, 174104.

(64) Wang, L.; Maxisch, T.; Ceder, G. Oxidation Energies of Transition Metal Oxides within the GGA+U Framework. Phys. Rev. B: Condens. Matter Mater. Phys. 2006, 73, 195107.
(65) Needs, R. J.; Towler, M. D.; Drummond, N. D.; Lopez Rios, P. Continuum Variational and Diffusion Quantum Monte Carlo Calculations. J. Phys.: Condens. Matter 2010, 22, 023201.

(66) Zen, A.; Sorella, S.; Gillan, M. J.; Michaelides, A.; Alfe, D. Boosting the Accuracy and Speed of Quantum Monte Carlo: Size Consistency and Time Step. Phys. Rev. B: Condens. Matter Mater. Phys. 2016, 93, 241118.

(67) Trail, J. R.; Needs, R. J. Smooth Relativistic Hartree-Fock Pseudopotentials for $\mathrm{H}$ to $\mathrm{Ba}$ and $\mathrm{Lu}$ to Hg. J. Chem. Phys. 2005, 122, 174109.

(68) See also http://www.tcm.phys.cam.ac.uk/ mdt26/casino manual_dir/casino_manual.pdf, Date of access: April 21, 2017.

(69) Sola, E.; Brodholt, J. P.; Alfe, D. Equation of State of Hexagonal Closed Packed Iron Under Earth's Core Conditions from Quantum Monte Carlo Calculations. Phys. Rev. B: Condens. Matter Mater. Phys. 2009, 79, 1-6.

(70) Casula, M.; Moroni, S.; Sorella, S.; Filippi, C. Size-Consistent Variational Approaches to Nonlocal Pseudopotentials: Standard and Lattice Regularized Diffusion Monte Carlo Methods Revisited. J. Chem. Phys. 2010, 132, 154113.

(71) Baroni, S.; Dal Corso, A.; De Gironcoli, S.; Giannozzi, P. PWSCF Package. http://www.pwscf.org/, Date of access: April 21, 2017.

(72) Alfe, D.; Gillan, M. J. Efficient Localized Basis Set for Quantum Monte Carlo Calculations on Condensed Matter. Phys. Rev. B: Condens. Matter Mater. Phys. 2004, 70, 1.

(73) Chiesa, S.; Ceperley, D. M.; Martin, R. M.; Holzmann, M. FiniteSize Error in Many-Body Simulations with Long-Range Interactions. Phys. Rev. Lett. 2006, 97, 076404.

(74) Fraser, L. M.; Foulkes, W. M. C.; Rajagopal, G.; Needs, R. J.; Kenny, S. D.; Williamson, A. J. Finite-Size Effects and Coulomb Interactions in Quantum Monte Carlo Calculations for Homogeneous Systems with Periodic Boundary Conditions. Phys. Rev. B: Condens. Matter Mater. Phys. 1996, 53, 1814-1832.

(75) Geier, S. J.; Mason, J. A.; Bloch, E. D.; Queen, W. L.; Hudson, M. R.; Brown, C. M.; Long, J. R. Selective Adsorption of Ethylene over Ethane and Propylene over Propane in the Metal-Organic Frameworks $\mathrm{M} 2($ dobdc) (M = Mg, Mn, Fe, Co, Ni, Zn). Chem. Sci. 2013, 4, 20542061.

(76) Sanz, R.; Martinez, F.; Orcajo, G.; Wojtas, L.; Briones, D. Synthesis of a Honeycomb-Like Cu-Based Metal-Organic Framework and its Carbon Dioxide Adsorption Behaviour. Dalton Trans. 2013, 42, 2392-2398.

(77) Cadot, S.; Veyre, L.; Luneau, D.; Farrusseng, D.; Alessandra Quadrelli, E. A Water-Based and High Space-Time Yield Synthetic Route to MOF Ni2(dhtp) and its Linker 2,5-dihydroxyterephthalic Acid. J. Mater. Chem. A 2014, 2, 17757-17763.

(78) McCusker, L. B.; Von Dreele, R. B.; Cox, D. E.; Louer, D.; Scardi, P. Rietveld Refinement Guidelines. J. Appl. Crystallogr. 1999, 32, 36-50.

(79) Larson, A. C.; Von Dreele, R. B. General Structure Anaylsis System (GSAS). Structure 2004, 748.

(80) Toby, B. H. EXPGUI, A Graphical User Interface for GSAS. J. Appl. Crystallogr. 2001, 34, 210-213.

(81) Wu, H.; Zhou, W.; Yildirim, T. High-Capacity Methane Storage in Metal-Organic Frameworks M2(dhtp): The Important Role of Open Metal Sites. J. Am. Chem. Soc. 2009, 131, 4995-5000.

(82) Lee, K.; Isley, W. C., III; Dzubak, A. L.; Verma, P.; Stoneburner, S. J.; Lin, L. C.; Howe, J. D.; Bloch, E. D.; Reed, D. A.; Hudson, M. R.; Brown, C. M.; Long, J. R.; Neaton, J. B.; Smit, B.; Cramer, C. J.; Truhlar, D. G.; Gagliardi, L. Design of a Metal-Organic Framework with Enhanced Back Bonding for Separation of N2 and CH4. J. Am. Chem. Soc. 2014, 136, 698-704.

(83) Momma, K.; Izumi, F. VESTA3 for Three-Dimensional Visualization of Crystal, Volumetric and Morphology Data. J. Appl. Crystallogr. 2011, 44, 1272-1276.

(84) Poloni, R.; Lee, K.; Berger, R. F.; Smit, B.; Neaton, J. B. Understanding Trends in CO2 Adsorption in Metal-Organic Frameworks with Open-Metal Sites. J. Phys. Chem. Lett. 2014, 5, 861-865.

(85) Piccini, G.; Sauer, J. Effect of Anharmonicity on Adsorption Thermodynamics. J. Chem. Theory Comput. 2014, 10, 2479-2487. 
(86) Piccini, G.; Alessio, M.; Sauer, J.; Zhi, Y.; Liu, Y.; Kolvenbach, R.; Jentys, A.; Lercher, J. A. Accurate Adsorption Thermodynamics of Small Alkanes in Zeolites. Ab Initio Theory and Experiment for H-Chabazite. J. Phys. Chem. C 2015, 119, 6128-6137.

(87) Al-Hamdani, Y. S.; Ma, M.; Alfè, D.; von Lilienfeld, O. A.; Michaelides, A. Communication: Water on Hexagonal Boron Nitride from Diffusion Monte Carlo. J. Chem. Phys. 2015, 142, 181101.

(88) Ma, J.; Michaelides, A.; Alfe, D.; Schimka, L.; Kresse, G.; Wang, E. Adsorption and Diffusion of Water on Graphene from First Principles. Phys. Rev. B: Condens. Matter Mater. Phys. 2011, 84, 33402.

(89) Queen, W. L.; Brown, C. M.; Britt, D. K.; Zajdel, P.; Hudson, M. R.; Yaghi, O. M. Site-Specific CO2 Adsorption and Zero Thermal Expansion in an Anisotropic Pore Network. J. Phys. Chem. C 2011, 115, 24915-24919.

(90) Liu, Y.; Kabbour, H.; Brown, C. M.; Neumann, D. A.; Ahn, C. C. Increasing the Density of Adsorbed Hydrogen with Coordinately Unsaturated Metal Centers in Metal-Organic Frameworks. Langmuir 2008, 24, 4772-4777.

(91) Bloch, E. D.; Hudson, M. R.; Mason, J. A.; Chavan, S.; Crocella, V.; Howe, J. D.; Lee, K.; Dzubak, A. L.; Queen, W. L.; Zadrozny, J. M.; Geier, S. J.; Lin, L. C.; Gagliardi, L.; Smit, B.; Neaton, J. B.; Bordiga, S.; Brown, C. M.; Long, J. R. Reversible CO Binding Enables Tunable CO/ $\mathrm{H} 2$ and $\mathrm{CO} / \mathrm{N} 2$ Separations in Metal-Organic Frameworks with Exposed Divalent Metal Cations. J. Am. Chem. Soc. 2014, 136, 1075261.

(92) Mercado, R.; Vlaisavljevich, B.; Lin, L.-C.; Lee, K.; Lee, Y.; Mason, J. A.; Xiao, D. J.; Gonzalez, M. I.; Kapelewski, M. T.; Neaton, J. B.; Smit, B. Force Field Development from Periodic Density Functional Theory Calculations for Gas Separation Applications Using Metal-Organic Frameworks. J. Phys. Chem. C 2016, 120, 12590-12604. 